\title{
Monte Carlo simulation of the nonadditive restricted primitive model of ionic fluids: Phase diagram and clustering
}

\author{
Riccardo Fanton:* \\ Dipartimento di Scienze dei Materiali e Nanosistemi, Università Ca' Foscari Venezia, \\ Calle Larga S. Marta DD2137, I-30123 Venezia, Italy \\ Giorgio Pastoređ \\ Dipartimento di Fisica dell' Università and IOM-CNR, Strada Costiera 11, 34151 Trieste, Italy
}

(Dated: March 1, 2022)

\begin{abstract}
We report an accurate Monte Carlo calculation of the phase diagram and clustering properties of the restricted primitive model with non-additive hard-sphere diameters. At high density the positively non-additive fluid shows more clustering than the additive model and the negatively non-additive fluid shows less clustering than the additive model, at low density the reverse scenario appears. A negative nonadditivity tends to favor the formation of neutrally charged clusters starting from the dipole. A positive nonadditivity favors the pairing of like ions at high density. The critical point of the gas-liquid phase transition moves at higher temperatures and higher densities for a negative nonadditivity and at lower temperatures and lower densities for a positive nonadditivity. The law of corresponding states does not seem to hold strictly. Our results can be used to interpret recent experimental works on room temperature ionic liquids.
\end{abstract}

PACS numbers: 68.43.Hn,61.20.Qg,64.70.pv,64.60.ah,64.70.F-,64.60.F-,64.75.Yz, Keywords: Monte Carlo simulation, Gibbs Ensemble Monte Carlo, Restricted Primitive Model, Non-Additive Hard-Spheres, Coexistence, Clustering, Radial distribution function, Structure factor

\section{INTRODUCTION}

Ionic soft matter [1] is a class of conventional condensed soft matter 2, 3] with prevailing contribution from electrostatics, in many cases crucially affecting its physical properties. Among the most popular representatives of such a class of materials are natural and synthetic saline environments, like aqueous and non-aqueous electrolyte solutions and molten salts as well as a variety of polyelectrolytes and colloidal suspensions. Equally well known are many biologically important proteins.

The simplest theoretical model for ionic colloidal suspensions is the Restricted Primitive Model (RPM) [4], a binary mixture of uniformly charged Hard-Spheres (HS) of diameter $\sigma$ : two species of opposite charge $\pm q$ and equal concentrations to ensure charge neutrality, moving in a medium of fixed dielectric constant $\epsilon$. The phase diagram properties of this model have been widely studied both through analytical theories [5- 17] and within computer experiments starting from the seminal works of Larsen 18] and Vel'Yaminov 19, 20], followed by the pioneering Gibbs Ensemble Monte Carlo (GEMC) calculation of Panagiotopoulos [21] and by other numerical simulations 22 29]. The more general primitive model (PM) with asymmetry in ion charge [30], in ion size 3133] and in both [32, 34] has also been studied.

From these studies emerged how, in the vapor phase, an important role is played by association and clustering. In an old paper [35] one of us studied a modified

\footnotetext{
* rfantoni@ts.infn.it

† pastore@ts.infn.it
}

RPM fluid where one allows for size nonadditivity particle diameters. Controlling the nonadditivity, it was suggested through the use of integral equation theories, that such a fluid might have a complex behavior due to the possible competition between clustering tendence due to the Coulomb interaction and demixing tendence due to entropic advantage driven by the nonadditivity. Thus, the nonadditivity of the hard-sphere diameters does not destroy the simplifying symmetry of the model but it enriches the properties of the pure RPM model making it a paradigm for the self-assembly of isotropic particles and a challenge to present day theories of fluids. In real systems, the degree of nonadditivity might be directly related to the anion-cation contact-pairing affinity 36] which in turn may be mediated by the solvent.

It is the purpose of this paper to reconsider such a model fluid from the point of view of accurate numerical experiments. In particular, we want to study the clustering properties of the fluid outside of the gas-liquid coexistence region. To this aim we first determined the gas-liquid coexistence curve through the Gibbs ensemble method after having studied semi-quantitatively how the coexistence region changes with the nonadditivity through a density distribution analysis in the canonical ensemble. This way we could be sure that our cluster analysis falls outside the coexistence region in all the cases studied. Clustering turns out to be greatly affected by the nonadditivity parameter. The most striking effect being the prevalence of neutrally charged clusters made up of an even number of particles in the negatively nonadditive fluid. When the nonadditivity allows complete overlap of the two species of particles the formation of a fluid of neutral hard-spheres of half the density is ex- 
pected and our simulation results clearly show this behavior. On the other hand, for a positive nonadditivity, it is known that the neutral HS mixture tends to demix the two species and the demixing critical density lowers as the nonadditivity increases [37]. We expect this property of the neutral system to have some interesting effect on the clustering properties of the charged fluid since demixing cannot occur in a binary charged system: The frustrated tendency to segregation of like particles and the reduced space available to the ions favors pairing of like ions and percolating clusters at high densities. To the best of our knowledge this is the first time that such a model fluid is studied with numerical simulations. Preliminary results from our analysis have been presented in a letter [38], here we extend that analysis and present for the first time the gas-liquid binodal of the fluid as a function of the nonadditivity parameter.

We think that the model fluid considered in this paper may be realized experimentally through a colloid-star polymer mixture where both species are charged [39] or by room temperature ionic liquids [40 43] as discussed in Section III 2 In particular in the latter systems liquidliquid binodals shifted above and below the one of the pure RPM are observed depending on the kind of solvent used. If on the one hand this can be ascribed to the different dielectric constant of the solvent [41], on the other hand it is clear that, depending on the kind of solvent, the anion-cation contact-pairing affinity may vary [36] and thus the different experimental ionic liquids should be more correctly described by comparison not just with the pure RPM but with the more realistic primitive model with the addition of either a positive or negative size nonadditivity.

The paper is organized as follows: in Section II the model for the fluid we want to study is described, in Section III the results from the numerical experiments are reported. These are divided in a cluster analysis in Section IIIA in an analysis of the radial distribution function and structure factor in Section ПI and in an analysis of the gas-liquid coexistence in Section IIIC Theoretical remarks on the clustering properties are presented in Section $[\mathrm{IV}$ and Section $\mathrm{V}$ is for final remarks.

\section{THE MODEL}

The model fluid we want to study is the restricted primitive model (RPM) of non-additive hard-spheres (NAHS). The RPM consists of $N / 2$ uniformly charged hard-spheres of species 1 of diameter $\sigma$ carrying a total charge $+q$ each and $N / 2$ uniformly charged hard-spheres of species 2 of the same diameter carrying a total charge $-q$ each. The spheres are moving in a dielectric continuum of dielectric constant $\epsilon$ independent on the thermodynamic state. The interaction between an ion of species $i$ and one of species $j$ a distance $r$ apart is given by

$$
\beta \phi_{i j}(r)=\left\{\begin{array}{ll}
+\infty & r \leq \sigma_{i j} \\
\frac{q_{i} q_{j}}{k_{B} T \epsilon r} & r>\sigma_{i j}
\end{array} \quad, \quad i, j=1,2,\right.
$$

where $\beta=1 / k_{B} T$ with $T$ the absolute temperature and $k_{B}$ the Boltzmann's constant, $q_{i}$ the charge of an ion of species $i$. The ions form a mixture of non-additive hardspheres, i.e.

$$
\sigma_{i j}=\left\{\begin{array}{ll}
\sigma & i=j \\
\sigma(1+\Delta) & i \neq j
\end{array}, \quad i, j=1,2,\right.
$$

with the nonadditivity parameter $\Delta>-1$. A thermodynamic state is completely specified by the reduced density $\rho^{*}=\rho \sigma^{3}=N \sigma^{3} / V$, where $V$ is the volume containing the fluid, the reduced temperature $T^{*}=k_{B} T \epsilon \sigma / q^{2}$ $\left(q^{2} /(\epsilon \sigma)\right.$ is taken as unit of energy), and the nonadditivity parameter $\Delta$. We will call $x_{1}=\rho_{-} / \rho=1 / 2$ and $x_{2}=\rho_{+} / \rho=1 / 2$ the anions and cations molar concentrations respectively.

\section{RESULTS}

In Fig. 1 we show the phase diagram of the pure RPM fluid, $\Delta=0$, as obtained from the Gibbs ensemble Monte Carlo method by Orkoulas et al. [24] and by us (see Section III 2 2). The thermodynamic points where we probe the fluid with our NVT Monte Carlo simulations are also shown as filled squares.

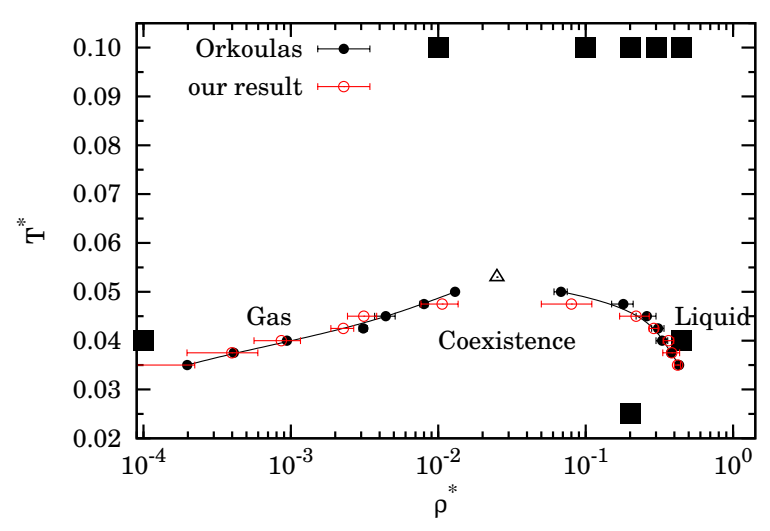

FIG. 1. (Color online) Diagram showing the gas-liquid coexistence curve of the RPM fluid from the Gibbs ensemble MC data of Ref. 24] (filled circles) and ours (open circles), the triangle being the critical point, and the points (filled squares) of the phase diagram where we run our NVT MC simulations.

In our canonical NVT Monte Carlo (MC) we study the fluid in a simulation box of volume $V=L^{3}$ with periodic boundary conditions. The long range of the $1 / r$ interaction is accounted for using an Ewald sum for the 
interacting energy in the periodic system [44]. The interaction energy per unit box for $\epsilon=1$ is calculated as

$$
\begin{aligned}
\mathcal{U}= & \sum_{\mu<\nu} \sum_{\mathbf{n}} q_{i_{\mu}} q_{j_{\nu}} \frac{\operatorname{erfc}\left(\kappa\left|\mathbf{r}_{\mu \nu}+L \mathbf{n}\right|\right)}{\left|\mathbf{r}_{\mu \nu}+L \mathbf{n}\right|} \\
& +\frac{2 \pi}{L^{3}} \sum_{\mathbf{k} \neq \mathbf{0}} \frac{e^{-(k / 2 \kappa)^{2}}}{k^{2}}\left|\rho_{\mathbf{k}}\right|^{2}-\frac{\kappa}{\sqrt{\pi}} \sum_{\mu} q_{i_{\mu}}^{2} \\
& -\frac{\pi}{2 \kappa^{2} L^{3}}\left(\sum_{\mu} q_{i_{\mu}}\right)^{2},
\end{aligned}
$$

where a Roman index with a Greek sub-index denotes the species of the particle labeled by the Greek subindex, $\mathbf{r}_{\mu \nu}=\mathbf{r}_{\nu}-\mathbf{r}_{\mu}$ with $\mathbf{r}_{\mu}$ the position of particle $\mu, \rho_{\mathbf{k}}=\sum_{\mu} q_{i_{\mu}} e^{-i \mathbf{k} \cdot \mathbf{r}_{\mu}}$, erfc denotes the complementary error function, $\mathbf{n}=\left(n_{x}, n_{y}, n_{z}\right)$ with $n_{x}, n_{y}, n_{k}=$ $0, \pm 1, \pm 2, \ldots$, and $\mathbf{k}=(2 \pi / L)\left(n_{x}, n_{y}, n_{z}\right)$ are reciprocal lattice vectors. The parameter $\kappa$ which governs the rate of convergence of the real space and reciprocal space contribution to the energy is taken to be $\kappa \sim 5 / L$. With this value of $\kappa$, the real space contribution can be restricted to the first term $\mathbf{n}=\mathbf{0}$ only. The reciprocal space term includes all $\mathbf{k}$ vectors such that $n_{x}^{2}+n_{y}^{2}+n_{z}^{2}<27$. The last term in Eq. (3.1) is zero for the RPM but it is important in the Gibbs ensemble simulation where a particle exchange between the two boxes can produce systems where there is an unequal number of positive and negative charges. Our choice for the interaction energy takes into account the fact that each charge has a uniform background of neutralizing opposite charge density.

In our $N V T$ MC simulations we used $N=100$ (except for the test of the size dependence of the clustering analysis where we considered up to 5000 particles), the acceptance ratio is kept, on average, close to $50 \%$ after a preliminary adjustment of the maximum particle displacement. We start from a simple cubic configuration of two crystals one made of species 1 and one made of species 2 juxtaposed in order to avoid overlaps at high densities. We need around $10^{5} \mathrm{MC}$ steps (MCS) in order to equilibrate the samples and $10^{6} \mathrm{MCS} /$ particle for the statistics.

\section{A. Cluster analysis}

During the simulation we perform a cluster analysis. After each $100 \mathrm{MCS}$ we determine the number $N_{n}$ of clusters made of $n$ particles, so that $\sum_{n} n N_{n}=N$. We assume [45] that a group of ions forms a cluster if the distance $r$, calculated using periodic boundary conditions, between a particle of species $i$ of the group and at least one other particle of species $j$ is less then some fixed value, i.e. $r<\sigma_{i j}+\delta^{c} \sigma$ where $\delta^{c}$ is a parameter 46]. In all our simulations we choose $\delta^{c}=0.1$ (in Ref. 25] a detailed study of the sensitivity of the clustering properties on this parameter is carried out for the pure RPM fluid). Then we take the average of these numbers $\left\langle N_{n}\right\rangle$.
Note that $Q_{n}=n\left\langle N_{n}\right\rangle / N$ gives the probability that a particle belongs to a cluster of size $n$. To establish a criterion for percolation we first find the clusters without employing periodic boundary conditions to calculate the distances, then we check whether, amongst the particles of any of these clusters, there are two which satisfy the cluster condition calculating the distances using periodic boundary conditions. Whenever we find one such cluster the cluster is percolating.

In Fig. 2 we show results of such analysis for the fluid at a temperature $T^{*}=0.1$ well above the critical temperature, $T_{c}^{*} \approx 0.05$, of the pure RPM [26, 28, 29]. In the insets we show a magnification of the region around $n=1$ from which the degree of dissociation [14, 17] $\alpha=\left\langle N_{1}\right\rangle / N$ can be read-off. In the figure we plot the cluster concentrations $\left\langle N_{n}\right\rangle / N$ as a function of the number of particles $n$ in the cluster. We plot $n$ from $n=1$ (isolated ions) up to $n=N$ (in this case all the particles of the fluid form one big percolating cluster). At $\rho^{*}=0.45$ both the pure RPM and the $\Delta=+0.3$ fluid form percolating clusters. Lowering the density we first reach a state, at $\rho^{*}=0.3$, where the negative nonadditivity gives the same clustering of RPM and the positive nonadditivity gives bigger clustering (still with percolating clusters), then a state, at $\rho^{*}=0.1$, where the positive nonadditivity gives the same clustering of RPM and the negative nonadditivity a bigger one, and finally a state $\rho^{*}=0.01,0.001$, at low densities where a negative nonadditivity increases the clustering over the RPM fluid and a positive nonadditivity diminishes it. Generally, at high densities we find percolating clusters in the fluids whereas these disappear at low densities even at a positive nonadditivity. Summarizing, in agreement with Ref. [35], we find, for the fixed values of $|\Delta|$, that: at high density and positive $\Delta$ we have more clustering than in the additive model since there is a smaller effective volume for particle, at high density and negative $\Delta$ we have less clustering than in the additive model because there is more effective volume for the particles, at low density and positive $\Delta$ we have less clustering than in the additive model due to the competition between tendency to demixing in the corresponding neutral mixture and tendency to local electroneutrality of the Coulombic systems, at low densities and negative $\Delta$ we have more clustering than in the additive model because neutral clusters are favored as shown in the next Section. We conclude that at high temperature and high density the negative nonadditivity gives lower clustering than RPM, lowering the temperature at constant density or lowering the density at constant temperature it gradually tends to gives higher clustering than RPM. On the contrary, at low density the positive nonadditivity gives lower clustering than RPM, increasing the density it gradually tends to give larger clustering than RPM.

We determine the size dependence of the curves shown in Fig. 2 and see that when we have no percolating clusters, for example the data at $T^{*}=0.1, \rho^{*}=0.3, \Delta=$ $0,-0.3$, the curves were unaffected by a choice of a higher 
number of particles, while when we have percolating clusters, for example the data at $T^{*}=0.1, \rho^{*}=0.3, \Delta=0.3$, the curve $\left(n,\left\langle N_{n}\right\rangle / N\right)$ changes with $N$. In these latter cases we find that a common curve is given by $\left(x,\left\langle N_{x}\right\rangle / N\right)$ with $x=n / N \in[0,1]$. Then, in order to satisfy the normalization condition, $1=\sum_{n} n\left(\left\langle N_{n}\right\rangle / N\right) \approx$ $\int d x x N^{2}\left(\left\langle N_{x}\right\rangle / N\right)$, we must have for two different sizes $N^{\prime}$ and $N^{\prime \prime}$ that $\left(\left\langle N_{x}\right\rangle / N^{\prime}\right) /\left(\left\langle N_{x}\right\rangle / N^{\prime \prime}\right) \approx\left(N^{\prime \prime} / N^{\prime}\right)^{2}$. We have no general recipe to when the former behavior is to be expected over the latter. We can only say that the first behavior is generally observed when we do not have percolating clusters whereas the second is present when we have percolating clusters. In Section IV we show that the size independent curves that we find when there are no percolating clusters can be fitted by $\left\langle N_{n}\right\rangle / N=a^{n} n^{b n} / n$ ! (see Eq. (4.4) with $z_{n}^{\text {intra }}$ obtained from an ideal cluster approximation) with $a$ and $b$ a positive fitting parameter. In Table 1 we show the fitting parameters $a$ and $b$ corresponding to the simulated cases.

TABLE I. Fitting parameters $a, b$ in the least square fit $\left\langle N_{n}\right\rangle / N=a^{n} n^{b n} / n$ ! for the simulation results of Fig. 2 without percolating clusters (and with the exclusion of the non smooth data at $\left.\rho^{*}=0.001\right)$. The reduced $\chi^{2}$ was around 0.5 with greater error approaching $n=1$. Also shown is the number of particles $n_{\max }$ in the biggest cluster formed in each simulation.

\begin{tabular}{|c|c||c|c|c|}
\hline$\rho^{*}$ & $\Delta$ & $a$ & $b$ & $n_{\max }$ \\
\hline 0.45 & -0.3 & $0.220(3)$ & $1.074(4)$ & 64 \\
0.3 & 0 & $0.197(4)$ & $1.084(6)$ & 45 \\
0.3 & -0.3 & $0.204(3)$ & $1.069(5)$ & 43 \\
0.2 & 0 & $0.206(7)$ & $1.00(1)$ & 23 \\
0.2 & +0.3 & $0.200(4)$ & $1.083(5)$ & 45 \\
0.2 & -0.3 & $0.204(7)$ & $1.04(1)$ & 31 \\
0.1 & 0 & $0.22(2)$ & $0.86(3)$ & 15 \\
0.1 & +0.3 & $0.16(1)$ & $1.01(4)$ & 19 \\
0.1 & -0.3 & $0.15(1)$ & $1.11(2)$ & 29 \\
0.01 & 0 & $0.41(7)$ & $0.1(1)$ & 8 \\
0.01 & +0.3 & $0.36(8)$ & $0.0(2)$ & 7 \\
0.01 & -0.3 & $0.23(4)$ & $0.72(7)$ & 12 \\
\hline
\end{tabular}

In Fig. 3 we show the clustering analysis at the thermodynamic state below the critical temperature of RPM $T^{*}=0.04$ in the gas phase, $\rho^{*}=5 \times 10^{-5}$, and in the liquid phase, $\rho^{*}=0.45$. We see how in the gas phase only the first few clusters are present in agreement with similar results found in Ref. [25] and for a negative nonadditivity the dipoles are clearly the preferred kind of clusters with the smallest degree of dissociation amongst the three fluids considered. In the liquid phase all three fluids have percolating clusters.

In Fig. 4 we show the clustering analysis for the fluid with $\Delta$ approaching -1 at $T^{*}=0.1$ and $\rho^{*}=0.45$. We see how letting $\Delta$ approach -1 this stabilizes the neutrally charged clusters and lowers the degree of dissociation. The first stable cluster is the dipole: the "overlap" of a positive and a negative sphere. These are dipoles of moment $q r_{12}$ with $r_{12}<\sigma\left(1+\Delta+\delta^{c}\right)$ which may lack a gas-liquid criticality [47]. We clearly have a transition from a conducting to an insulating phase as $\Delta$ goes from 0 to -1 .

In Fig. 5 we show a snapshot of the equilibrated fluid at $T^{*}=0.1, \rho^{*}=0.45$ and $\Delta=-0.9$ from which one can see the formation of the dipoles. We expect that in the limiting case of $\Delta=-1$ the fluid we obtain is well reproduced by hard-spheres at half the density. This is confirmed by a comparison of the like radial distribution functions with the one of the hard-spheres even if the $\Delta=-1$ fluid simulation rapidly slows down into the frozen configuration of the overlapping anions and cations. In order to overcome this problem one should alternate single particle moves to cluster moves where one moves the center of mass of the neutrally charged pairs.

\section{B. Radial distribution function and structure factor}

In Figs. 6 6 8 we show the partial radial distribution functions $(\mathrm{RDF}), g_{i j}(r)=\left\langle\sum_{\mu \nu}^{\prime} \delta\left(\mathbf{r}+\mathbf{r}_{\nu}^{j}-\mathbf{r}_{\mu}^{i}\right)\right\rangle / N \rho x_{i} x_{j}$, where $\mathbf{r}_{\mu}^{i}$ denote the position of particle $\mu$ of species $i$ and the prime over the sum indicates that the terms $\mu=$ $\nu$ when $i=j$ are omitted, and the total RDF, $g_{\text {tot }}=$ $\sum_{i, j=1}^{2} g_{i j} x_{i} x_{j}$, of the three fluids $\Delta=0, \pm 0.3$ at the thermodynamic states $T^{*}=0.1, \rho^{*}=0.01,0.1$ and $T^{*}=$ $0.04, \rho^{*}=0.45$. Of course, the restrictions $x_{1}=x_{2}$ and $\sigma_{11}=\sigma_{22}$ imply $g_{11}=g_{22}$. In the simulations we use $N=100$.

From Fig. 6] we see how the contact value of the like RDF in the $\Delta=-0.3$ case is higher than in the additive case and in the $\Delta=+0.3$ case is lower than in the additive case. The contact value of the unlike RDF is highest for negative nonadditivity indicating the tendency to form cation-anion pairs.

From Fig. 7 we see again the same behaviors of the contact values of the like and unlike RDF. In the negatively additive case we begin to see an alternation of distribution of oppositely charged shells of ions around a reference ion.

From Fig. 8 we see how at this high density the contact value of the like RDF is highest in the $\Delta=-0.3$ case but in the $\Delta=+0.3$ case is still higher than in the additive case. At $\Delta=-0.3$ we see clearly the formation of a second peak in the unlike RDF around $2+\Delta$ and the expected alternation between the peaks of the like RDF with the ones of the unlike RDF also present in the additive case. This alternation is not present in the positively non-additive case indicating now the tendency of like particles to cluster on a microscopic scale: like particles penetrate inside the shell of unlike particles around a given reference ion. The contact value of the unlike RDF is highest for negative nonadditivity indicating the tendency to form cation-anion pairs.

In Fig. 9] we show the Bhatia-Thornton [48] struc- 

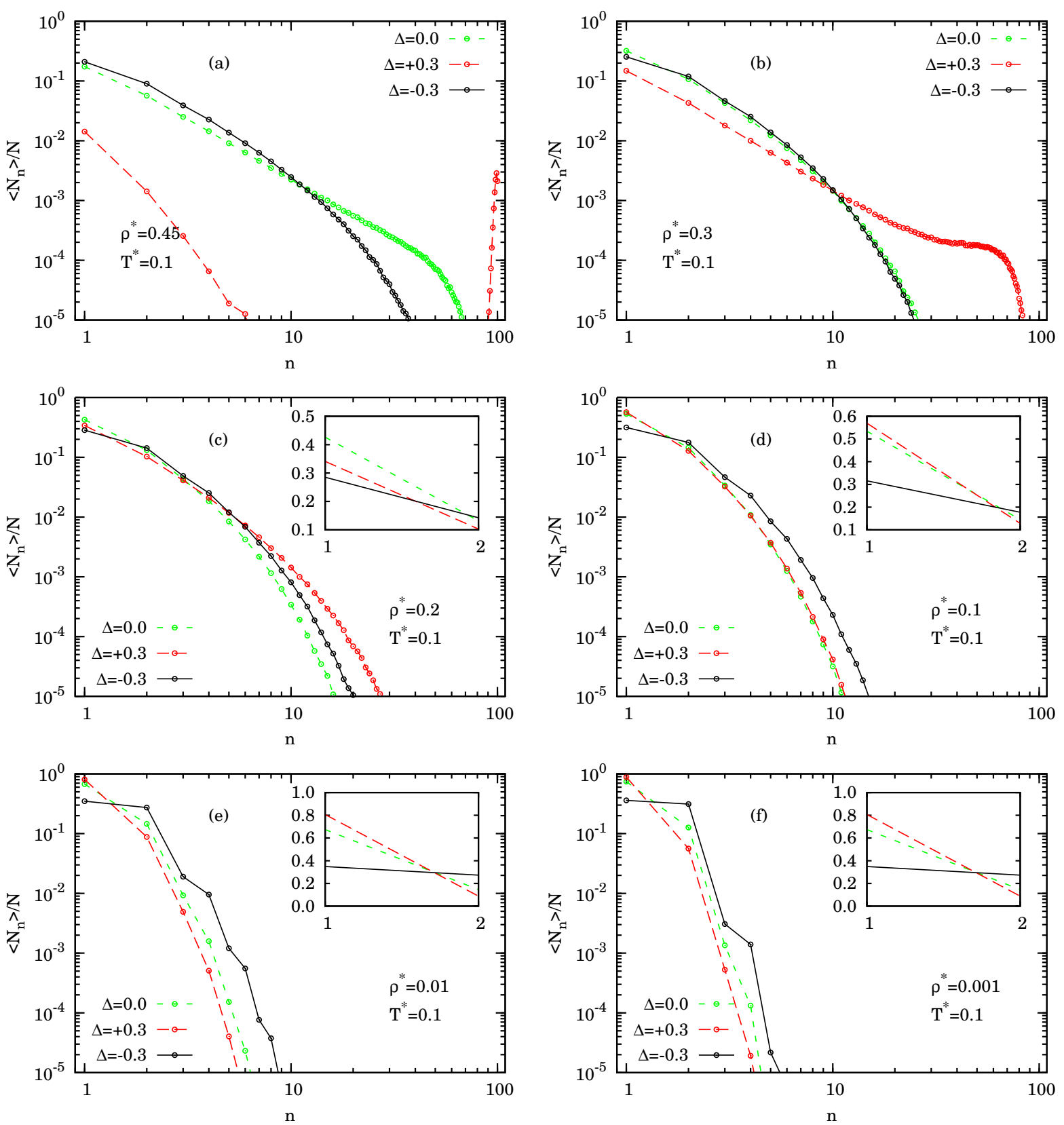

FIG. 2. (Color online) Clustering properties of the fluid at $T^{*}=0.1$ at various values of nonadditivity. $N_{n}$ are the number of clusters made of $n$ particles. In the MC simulations we used $N=100$ particles and a number of $\mathrm{MCS}=1 \times 10^{7}$. The panels are ordered (left to right, top to bottom) in order of decreasing density $\rho^{*}=0.45,0.3,0.2,0.1,0.01$, and 0.001 for panels (a), (b), (c), (d), (e), and (f) respectively. The insets allows to read-off the degree of dissociation.

ture factors $S_{N N}=\left[S_{11}+S_{22}+2 S_{12}\right] / 2$ and $S_{Q Q}=$ $\left[S_{11}+S_{22}-2 S_{12}\right] / 2$ where $S_{i j}(k)=\left\langle\rho_{\mathbf{k}}^{i} \rho_{-\mathbf{k}}^{j}\right\rangle / N \sqrt{x_{i} x_{j}}$ are the partial structure factors and $\rho_{\mathbf{k}}^{i}=\sum_{\mu} \exp \left(-i \mathbf{k} \cdot \mathbf{r}_{\mu}^{i}\right)$ is the Fourier transform of the microscopic density of particles of species $i$. In the figure we chose the same thermodynamic state and nonadditivity considered in Fig. 1 of Ref. [35]. The positive nonadditivity case has percolating clusters. From the figure we see that the charge-charge structure factor $S_{Q Q}$ tends to zero at $k=0$ a conse- quence of electroneutrality in charged systems [4] which suppresses long-wavelength fluctuations. In order to enforce this condition the structure factor needs to develop a peak at small $k$ which reflects an essentially alternating distribution of oppositely charged shell of ions around a reference ion. This type of short range order is an indication of the tendency to cluster. From the figure we see that at high density the positive non-additive fluid tends to cluster more than the additive fluid and the negative 

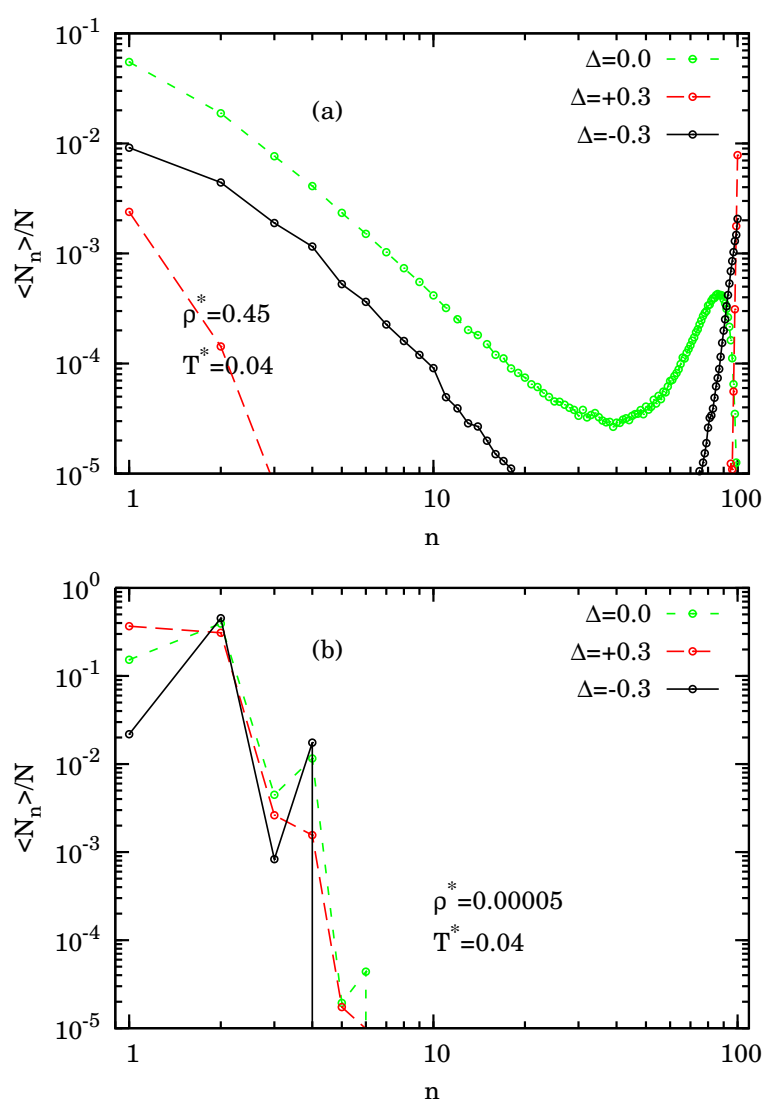

FIG. 3. (Color online) Clustering properties of the fluid at $T^{*}=0.04$ and $\rho^{*}=0.45,5 \times 10^{-5}$, for panels (a) and (b) respectively, at various values of nonadditivity. $N_{n}$ are the number of clusters made of $n$ particles. In the MC simulations we used $N=100$ particles and a number of $\mathrm{MCS}=1 \times 10^{7}$.

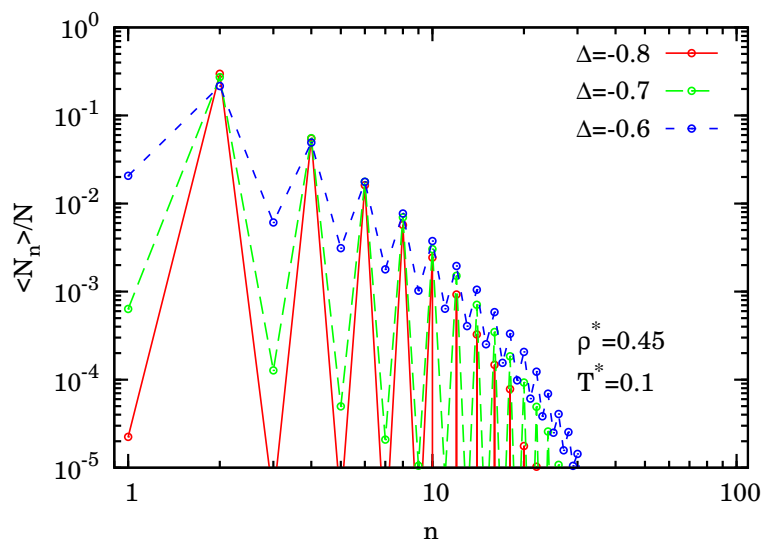

FIG. 4. (Color online) Clustering properties of the fluid at $T^{*}=0.1$ and $\rho^{*}=0.45$ at various values of negative nonadditivity approaching $-1 . N_{n}$ are the number of clusters made of $n$ particles. In the MC simulations we used $N=100$ particles and a number of $\mathrm{MCS}=5 \times 10^{7}$.

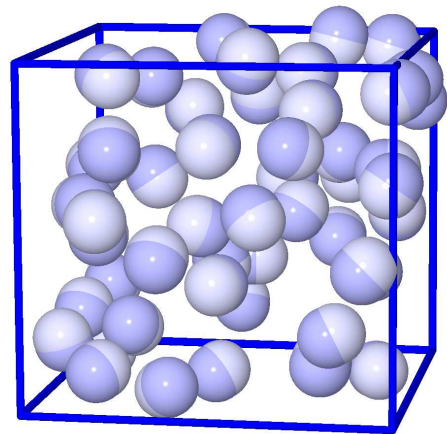

FIG. 5. (Color online) Snapshot of the fluid at $T^{*}=0.1, \rho^{*}=$ 0.45 and $\Delta=-0.9$ showing the formation of the dipoles.

non-additive fluid tends to cluster less than the additive fluid, in agreement with the results presented in the previous Section. With regard to the number-number structure factor $S_{N N}$ we see that as the nonadditivity decreases the isothermal compressibility $S_{N N}(0)$ (see the appendix of Ref. [48, 49]) increases and the short range order is reduced.

In Table $\amalg$ we report the excess internal energy per particle $U^{\mathrm{ex}} / N=\epsilon \sigma\langle\mathcal{U}\rangle / N q^{2}$, the compressibility factor $Z=\beta P / \rho$, and the total clusters concentration $\sum_{n}\left\langle N_{n}\right\rangle / N$ for the cases simulated. The compressibility factor is calculated according to the virial theorem

$$
\begin{aligned}
Z= & 1+\frac{U^{\mathrm{ex}}}{3 N T^{*}} \\
& +\frac{\pi \rho^{*}}{3}\left[g_{11}(\sigma)+(1+\Delta)^{3} g_{12}(\sigma(1+\Delta))\right] .
\end{aligned}
$$

If the clusters do not interact, as in the independent cluster model (ICM) of Gillan [7], one should have $Z^{\mathrm{ICM}}=$ $\sum_{n}\left\langle N_{n}\right\rangle / N$. From Table is never satisfied in the cases considered.

\section{Gas-liquid coexistence}

An important question we try to answer is how the gasliquid coexistence curve of the pure RPM fluid changes upon switching on of the nonadditivity parameter. To this aim we first perform a density distribution analysis within the $N V T$ ensemble which allows us to easily extract a semi-quantitative result and then we use the Gibbs ensemble technique for a careful quantitative determination of the binodals. 


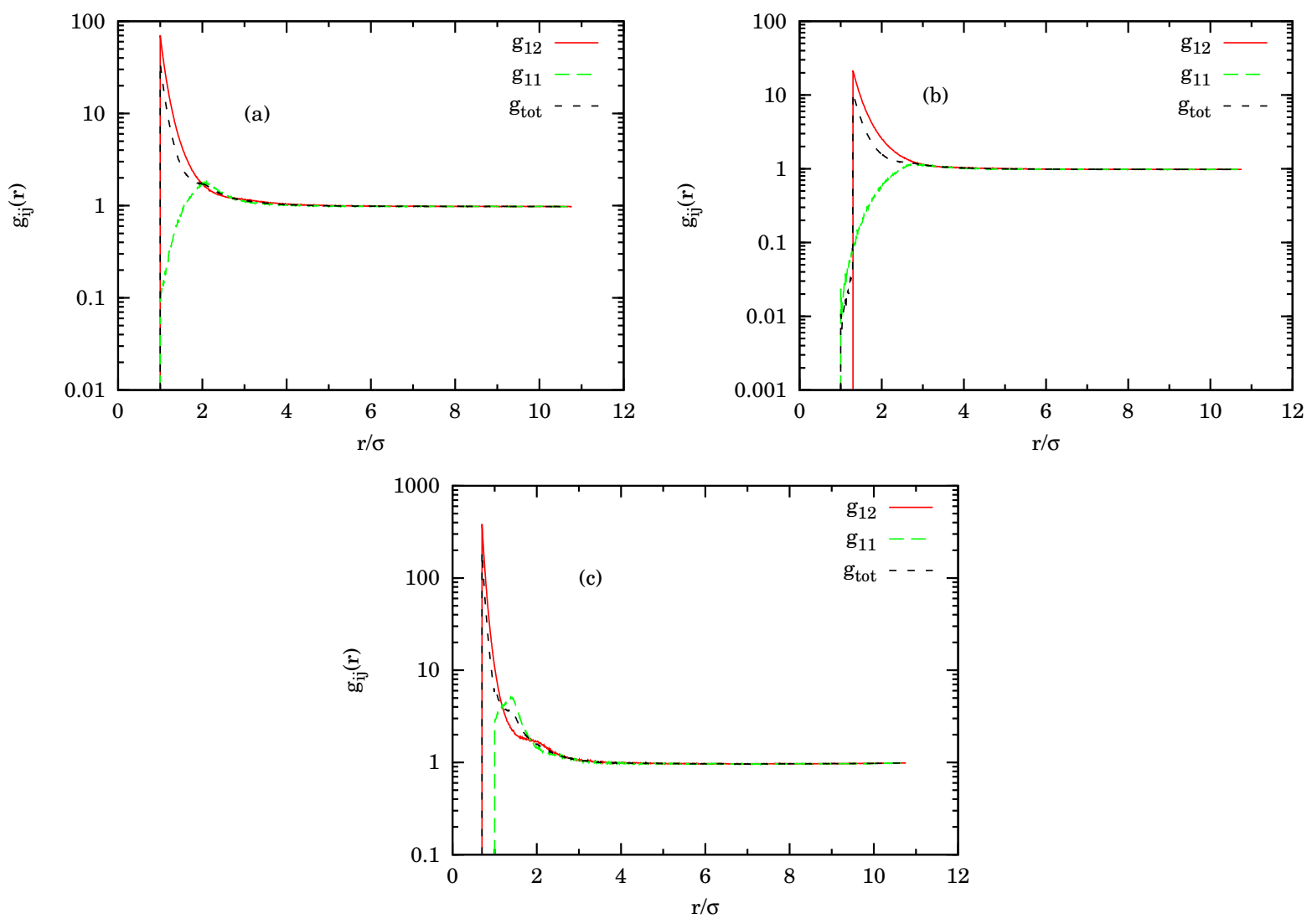

FIG. 6. (Color online) Partial and total RDF in the simulations at $\rho^{*}=0.01, T^{*}=0.1$, and $\Delta=0$ (panel (a)), $\Delta=0.3$ (panel (b)),$\Delta=-0.3$ (panel (c)). The reduced excess internal energy per particle of the fluid was in the three cases $U^{\text {ex }} / N=-0.3924(1),-0.29120(7),-0.6339(1)$ respectively.

\section{The density distribution approach}

Sufficiently close to the critical point we determine how semi-quantitatively the behavior of the gas-liquid coexistence region changes by switching on a negative or a positive nonadditivity. To this aim we divide the simulation box into $m^{3}$ cubes of side $L_{c}=L / m$ and register, as the run progresses, the density inside each cell $\rho_{i}=\mathcal{N}_{i} / L_{c}^{3}$, where $\mathcal{N}_{i}$ is the number of particles inside the $i$ th cell so that $\sum_{i=1}^{m^{3}} \mathcal{N}_{i}=N$. Then we calculate the density distribution function [50] $P_{m}(\rho)=\sum_{i=1}^{m^{3}} P_{m}\left(\rho_{i}\right) / m^{3}$, where $P_{m}\left(\rho_{i}\right)$ is the distribution function for the $i$ th cell, with $\int P_{m}(\rho) d \rho=1$. Above the critical temperature the density probability distribution function can be described by a Gaussian centered at the simulation density whereas below it becomes bimodal with two peaks one centered at the gas density and one at the liquid density.

We start from an initial configuration of particles of random species placed on a simple cubic lattice. We equilibrate (melt) the fluid for $10^{6} \mathrm{MCS} /$ particle. We then sample the distribution function every 10 MCS. To allow the particles to diffuse out of the cells we choose the subdivision of the simulation box in cells with a random displacement $\mathbf{r}=\left(r_{x}, r_{y}, r_{z}\right)$ with $r_{x}, r_{y}, r_{z} \in[0, L]$. This procedure turned out to greatly enhance the efficiency of the determination of the cell density distribution. And we measure the distribution function on runs of $1 \times 10^{6}$ MCS/particle.

Choosing $m=2$ and $N=100$ the results for the fluid at a temperature $T^{*}=0.025$ above the triple point of the RPM [51], a density $\rho^{*}=0.2$ well within the coexistence region of the pure RPM fluid, and $\Delta=0, \pm \mathcal{D}$ with $\mathcal{D}=10^{-1}, 10^{-2}, 5 \times 10^{-2}$ are shown in Fig. 10. In this case the minimum density that can be registered is $1 / L_{c}^{3}=0.2 \times 8 / 100=0.016$. We see that the pure RPM fluid shows a density distribution function with two peaks: The first one, which lies below the minimum density (and is not visible in our data), at approximately the low density of the gas phase and the second one at approximately the high density of the liquid phase around a reduced density of 0.3 . At $\mathcal{D}=10^{-2}$ the positions of the peaks are roughly the same to the pure RPM. At $\mathcal{D}=5 \times 10^{-2}$ the density of the liquid peak in the negatively non-additive fluid is higher than the one of the pure RPM whereas the positively non-additive fluid has a gas peak, now visible, at higher density than the pure RPM and a liquid peak at lower density than the pure 

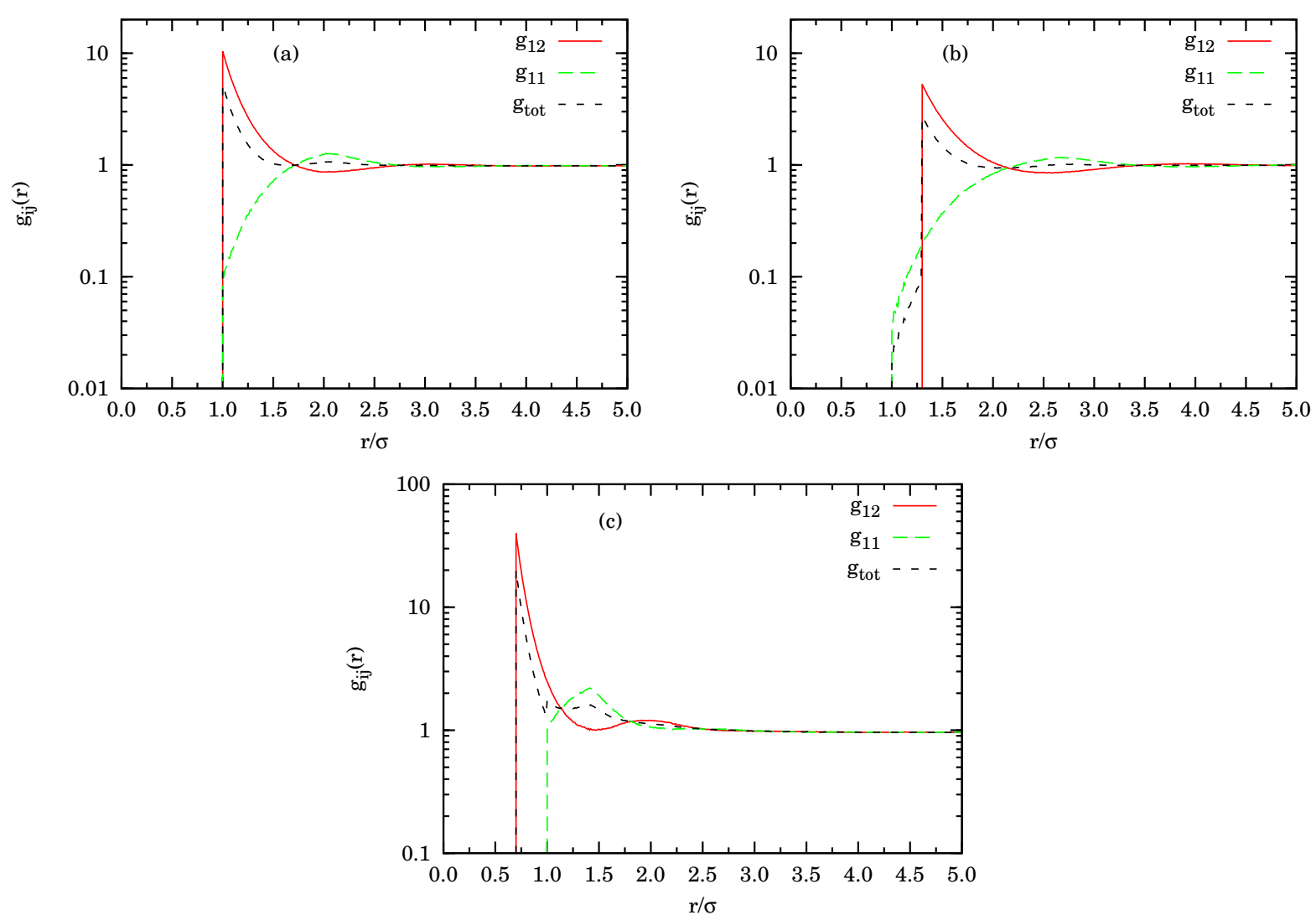

FIG. 7. (Color online) Same as Fig. [6 at $\rho^{*}=0.1$ and $T^{*}=0.1$. The reduced excess internal energy per particle of the fluid was in the three cases $U^{\text {ex }} / N=-0.50589(8),-0.41208(6),-0.7179(1)$ respectively.

RPM. At $\mathcal{D}=10^{-1}$ this separation tends to increase: In the positively non-additive model the critical temperature is too close to 0.025 and the bimodal is degenerate into a curve with a single peak centered on the simulation density 0.2 whereas in the negatively non-additive fluid the liquid peak is changed into a broad tail extending up to a density of 0.8 . This findings suggest that at a given temperature the width of the coexistence region, relative to the one of the pure RPM, tends to increase for the negatively non-additive model and to decrease for the positively non-additive model. This result is made more clear and precise in the following Section where we present our Gibbs Ensemble Monte Carlo calculation.

\section{Gibbs ensemble analysis}

In order to quantitatively determine the gas-liquid coexistence line of our fluid we use the Gibbs ensemble MC (GEMC) technique [52], starting from the pure RPM one and gradually switching on the nonadditivity. Here, we are not interested in the behavior really close to the critical point but rather in the shape of the binodal curve and how it moves as a function of $\Delta$.

The GEMC method of Panagiotopoulos is now widely adopted as a standard method for calculating phase equilibria from molecular simulations. According to this method, the simulation is performed in two boxes containing the coexisting phases. Equilibration in each phase is guaranteed by moving particles. Equality of pressures is satisfied in a statistical sense by expanding the volume of one of the boxes and contracting the volume of the other. Chemical potentials are equalized by transferring particles from one box to the other. Like the first simulations for the RPM performed by Panagiotopoulos 21] we use single ion transfer by introducing a background charge density to assure charge neutrality at all times during the run. This way, the system remains overall neutral, but the modified model is similar to a two component plasma and in a strict sense different from the original RPM model which assumes a zero charge density for the background. To overcome the electroneutrality problem Orkoulas et al. 24] considered pair transfers.

In the GEMC run we have at each step a probability $a_{p} /\left(a_{p}+a_{v}+a_{s}\right)$ for a particle random displacement, $a_{v} /\left(a_{p}+a_{v}+a_{s}\right)$ for a volume change, and $a_{s} /\left(a_{p}+a_{v}+a_{s}\right)$ for a particle swap move between the gas and the liquid box. We generally choose $a_{p}=1, a_{v}=1 / 10$, and $a_{s}=1$. The maximum particle displacement is kept 

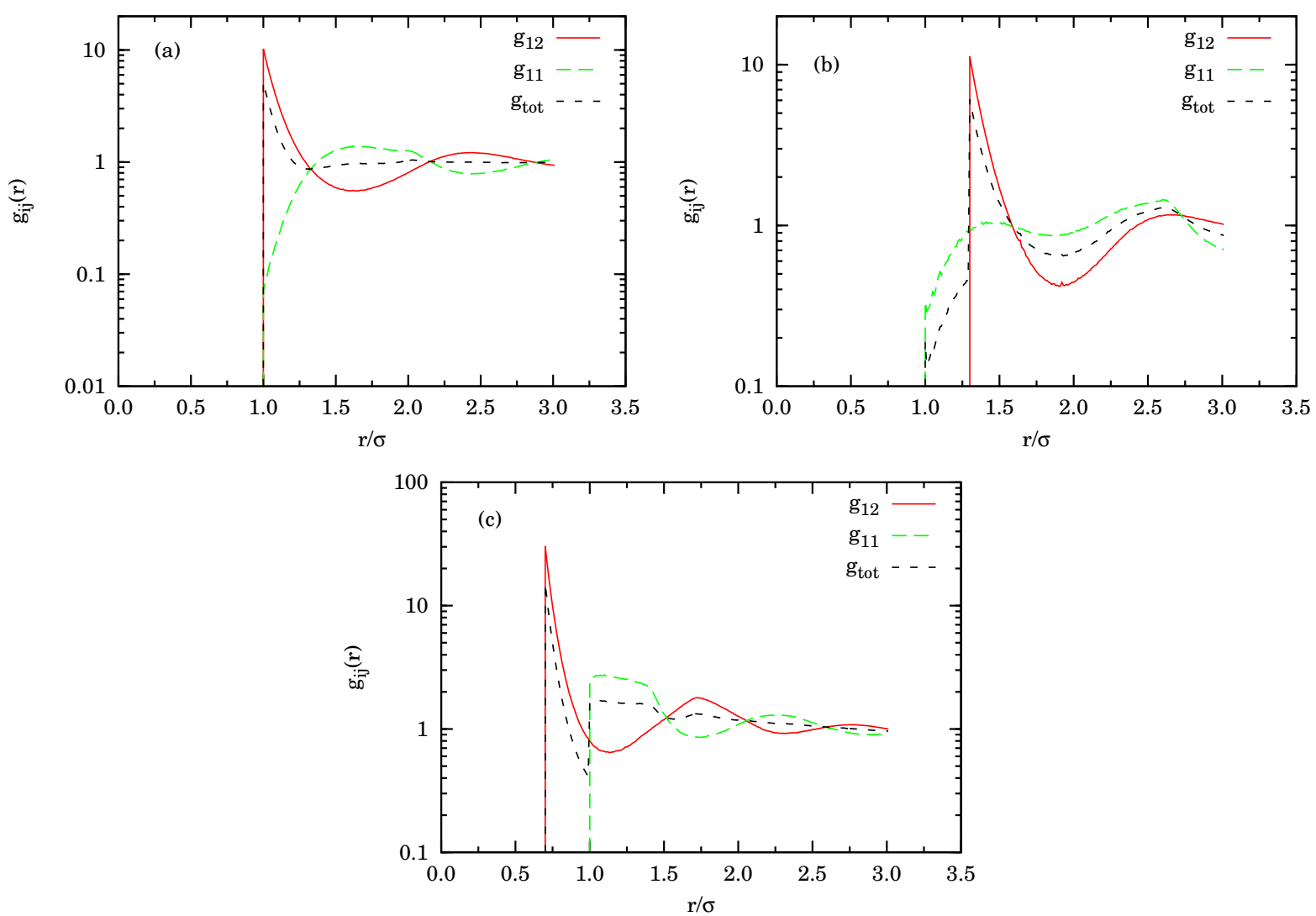

FIG. 8. (Color online) Same as Fig. [6 at $\rho^{*}=0.45$ and $T^{*}=0.04$. The reduced excess internal energy per particle of the fluid was in the three cases $U^{\text {ex }} / N=-0.69094(4),-0.55242(5),-0.96144(7)$ respectively.
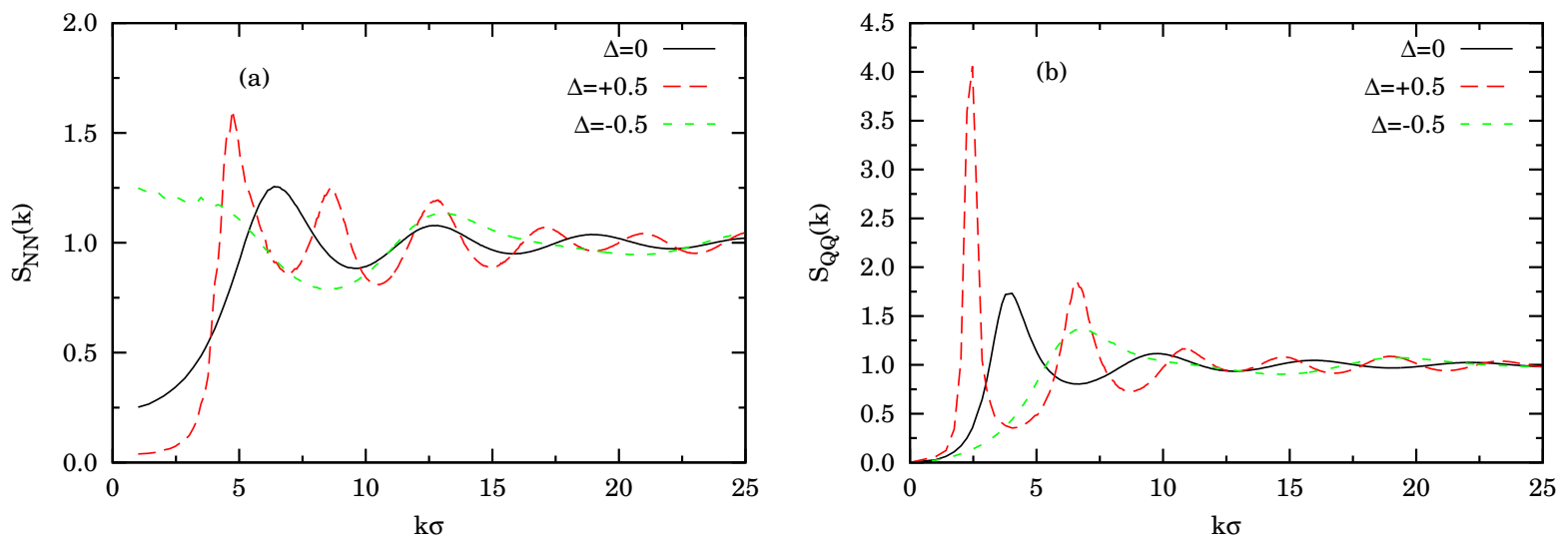

FIG. 9. (Color online) Bhatia-Thornton structure factors $S_{N N}(k)$ (panel (a)) and $S_{Q Q}(k)\left(\right.$ panel (b)) for $\rho^{*}=0.41253, T^{*}=$ 0.12 , and $\Delta=0, \pm 0.5$ as in Fig. 1 of Ref. 35]. Note that our abscissa has to be divided by 1.2 in order to compare with the units used in such reference.

equal to $L_{i} / 1000$ where $L_{i}$ is the side of the $i$ th box with $i=1,2$. Regarding the volume changes, following Ref. [52] we perform a random walk in $\ln \left[V_{1} / V_{2}\right]$, with $V_{i}$ the volume of the $i$ th box choosing a maximum volume displacement of $1 \%-10 \%$. Volume moves are computation- ally the cheapest since the energy scales with the length of the box with inverse proportionality. We generally use a total number of $N=100$ particles except close to the critical point where it proves necessary to increase the number of particles in order to avoid large fluctuations 

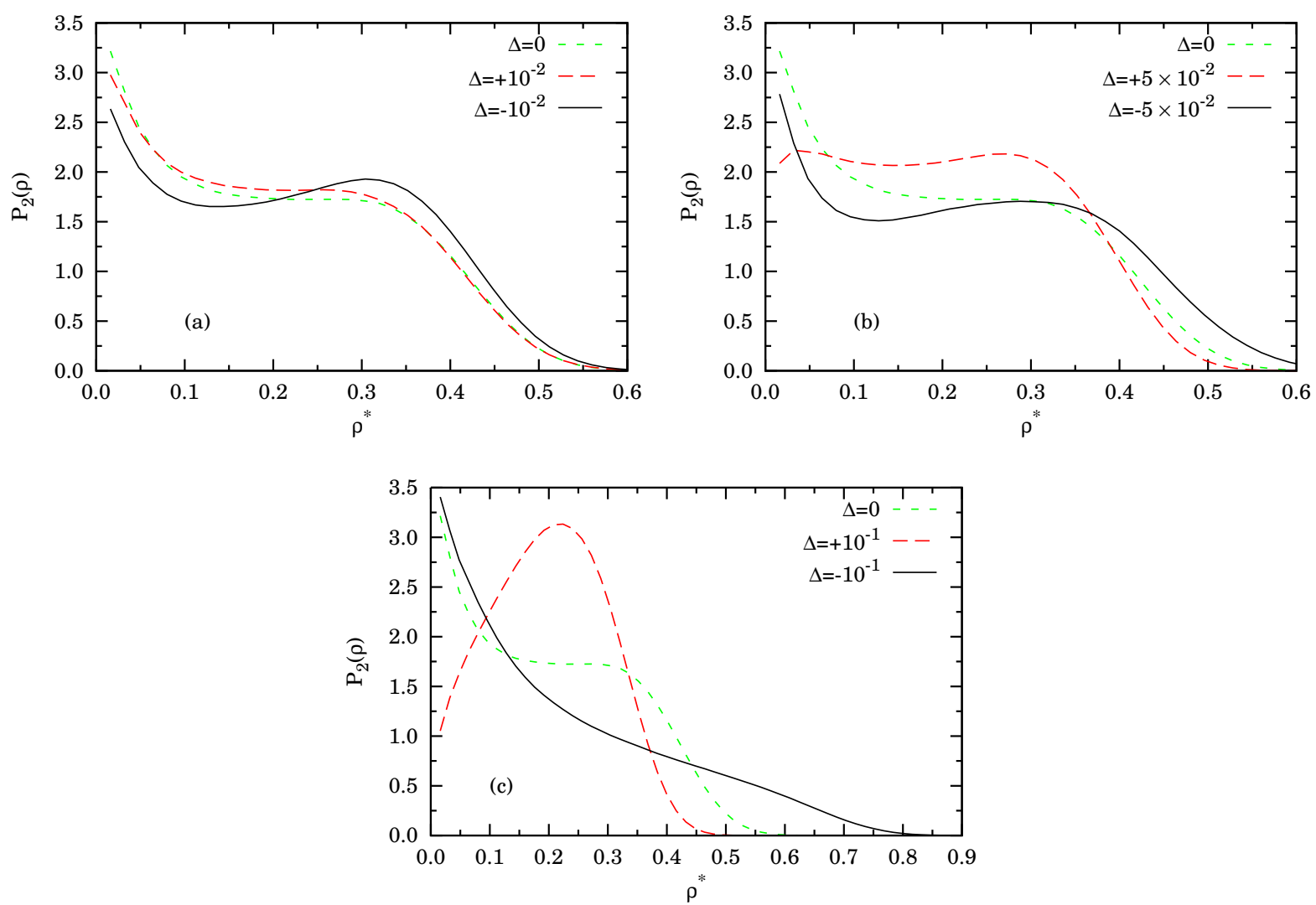

FIG. 10. (Color online) Cell density distribution function for the fluid at $T^{*}=0.025, \rho^{*}=0.2$ and $\Delta=0, \pm \mathcal{D}$ with $\mathcal{D}=$ $10^{-2}, 5 \times 10^{-2}, 10^{-1}$ in panels (a), (b), and (c) respectively. We used $N=100$ and $m=2$ with $1 \times 10^{6} \mathrm{MCS} / \mathrm{particle}$.

in the two densities. We use 10 - 40 million MCS for the equilibration and 100-200 million MCS for the production.

The results are summarized in Table 【II and Fig. 11 Note that since we get the same coexistence curve of Orkoulas et al. 24] for the pure RPM, as Fig. 10 clearly shows, we consider as equivalent, at the present level of accuracy, our procedure, employing single neutralized particle transfers, and the one of Orkoulas, where pair particle transfers between the two boxes are used. This can be justified by observing that the fluctuations of charge in the various statistical physics ensembles are expected to decay to zero with the system size and we empirically find that in our case they are already practically irrelevant. The only relevant difference we observe with respect to the calculation of Orkoulas is the fact that in our case there is a much more considerable emptying of the gas box at low temperatures which may have some effect in the point at the lowest temperature. We do not carry out a systematic study of possible system size dependence of the results but, for the pure RPM, we repeat the calculation at $T^{*}=0.045$ and 0.0475 for two different system sizes with the largest being $N=370$. The comparison suggests that the critical point tends to shift slightly at higher temperatures upon a system size increase but far away from the critical point the coexistence curve is not affected appreciably by the system size. However, we stress that an accurate study of critical properties of the present model is beyond the scope of this work.

From the figure we can see clearly the trend: a positive nonadditivity tends to lower the critical temperature whereas a negative one tends to push the binodal to higher temperatures. This is in agreement with the findings from the density distribution analysis previously presented. It is well known that RPM condensation is almost identical to that of charged hard dumbbells, underlining the fact that the vapor is essentially already fully associated into dimers and higher neutral clusters, and that the liquid structure and thermodynamics are only weakly perturbed by fusing ions together. Hence, if one imagines cooling down on the critical isochore, we can say that the critical point is reached when ion association is complete and then it becomes convenient for the system to phase separate. With positive nonadditivity, ion association is less favorable and the critical temperature must go down (association is complete only at lower temperatures); with negative nonadditivity, ion association is more favorable, and the critical temperature must go up (association is complete already at higher temper- 
TABLE II. Excess internal energy per particle $U^{\text {ex }}=$ $\epsilon \sigma\langle\mathcal{U}\rangle / q^{2}$, compressibility factor $Z=\beta P / \rho$, and total clusters concentration $\sum_{n}\left\langle N_{n}\right\rangle / N$ for the cases simulated.

\begin{tabular}{|c|c|c||c|c|c|}
\hline$T^{*}$ & $\rho^{*}$ & $\Delta$ & $-U^{\mathrm{ex}} / N$ & $Z-U^{\mathrm{ex}} / 3 N T^{*}$ & $\sum_{n}\left\langle N_{n}\right\rangle / N$ \\
\hline 0.1 & 0.45 & 0 & $0.62711(9)$ & $3.764(5)$ & 0.317 \\
0.1 & 0.45 & +0.3 & $0.46212(9)$ & $9.16(1)$ & 0.026 \\
0.1 & 0.45 & -0.3 & $0.81357(9)$ & $3.019(3)$ & 0.410 \\
\hline 0.1 & 0.3 & 0 & $0.58827(6)$ & $2.869(3)$ & 0.528 \\
0.1 & 0.3 & +0.3 & $0.47493(7)$ & $4.837(6)$ & 0.255 \\
0.1 & 0.3 & -0.3 & $0.7814(1)$ & $2.797(3)$ & 0.483 \\
\hline 0.1 & 0.2 & 0 & $0.55390(6)$ & $2.445(2)$ & 0.637 \\
0.1 & 0.2 & +0.3 & $0.45639(6)$ & $3.231(3)$ & 0.540 \\
0.1 & 0.2 & -0.3 & $0.75483(9)$ & $2.657(3)$ & 0.530 \\
\hline 0.1 & 0.1 & 0 & $0.50589(8)$ & $2.098(2)$ & 0.730 \\
0.1 & 0.1 & +0.3 & $0.41208(6)$ & $2.218(2)$ & 0.747 \\
0.1 & 0.1 & -0.3 & $0.7179(1)$ & $2.539(3)$ & 0.579 \\
\hline 0.1 & 0.01 & 0 & $0.3924(1)$ & $1.7373(8)$ & 0.830 \\
0.1 & 0.01 & +0.3 & $0.29120(7)$ & $1.493(3)$ & 0.900 \\
0.1 & 0.01 & -0.3 & $0.6339(1)$ & $2.409(2)$ & 0.652 \\
\hline 0.1 & 0.001 & 0 & $0.3076(1)$ & $1.582(1)$ & 0.870 \\
0.1 & 0.001 & +0.3 & $0.1971(1)$ & $1.2962(6)$ & 0.943 \\
0.1 & 0.001 & -0.3 & $0.5992(1)$ & $2.355(2)$ & 0.677 \\
\hline 0.04 & 0.45 & 0 & $0.69094(4)$ & $5.863(8)$ & 0.104 \\
0.04 & 0.45 & +0.3 & $0.55242(5)$ & $12.83(2)$ & 0.012 \\
0.04 & 0.45 & -0.3 & $0.96144(7)$ & $7.09(1)$ & 0.028 \\
\hline 0.04 & $5 \times 10^{-5}$ & 0 & $0.48804(2)$ & $4.112(1)$ & 0.563 \\
0.04 & $5 \times 10^{-5}$ & +0.3 & $0.35342(2)$ & $3.254(1)$ & 0.681 \\
0.04 & $5 \times 10^{-5}$ & -0.3 & $0.69764(1)$ & $5.230(2)$ & 0.493 \\
\hline
\end{tabular}

atures).

In order to determine the critical point $\left(T_{c}^{*}, \rho_{c}^{*}\right)$ we empirically fit the binodals using the "diameters" $\left(\rho_{g}^{*}+\right.$ $\left.\rho_{l}^{*}\right) / 2$ equation 53 ]

$$
\begin{aligned}
\frac{\rho_{g}^{*}+\rho_{l}^{*}}{2}= & \rho_{c}^{*}+A\left|T^{*}-T_{c}^{*}\right|+C\left|T^{*}-T_{c}^{*}\right|^{2 \beta_{I}} \\
& +D\left|T^{*}-T_{c}^{*}\right|^{1-\alpha_{I}},
\end{aligned}
$$

and the form of the Wegner expansion [53, 54] for the width of the coexistence curve

$$
\begin{aligned}
\rho_{l}^{*}-\rho_{g}^{*}= & B\left|T^{*}-T_{c}^{*}\right|^{\beta_{I}}+B_{1}\left|T^{*}-T_{c}^{*}\right|^{\beta_{I}+\Delta_{I}} \\
& +B_{2}\left|T^{*}-T_{c}^{*}\right|^{\beta_{I}+2 \Delta_{I}}
\end{aligned}
$$

where $A, C, D$, and $B, B_{1}, B_{2}$ are coefficients which we take as fitting parameters as well as $\rho_{c}^{*}, T_{c}^{*}$. We stress that our data do not extend sufficiently close to the critical region to allow quantitative estimates of critical exponents and non universal quantities, still we used the above functional forms as convenient fitting formulae, able to capture the typical flatness of the fluid coexistence curves [52]. The pure RPM is believed [28, 55 57] to belong to the three-dimensional Ising universality class so we choose $\beta_{I}=0.325, \alpha_{I}=0.11$, and $\Delta_{I}=0.51$. We are then able to fit the pure RPM case, $\Delta=0$, for which we find the critical point at $\rho_{c}^{*}=0.0319$ and $T_{c}^{*}=0.0476$, the RPM with positive nonadditivity, $\Delta=+0.1$, for which the critical point is found at

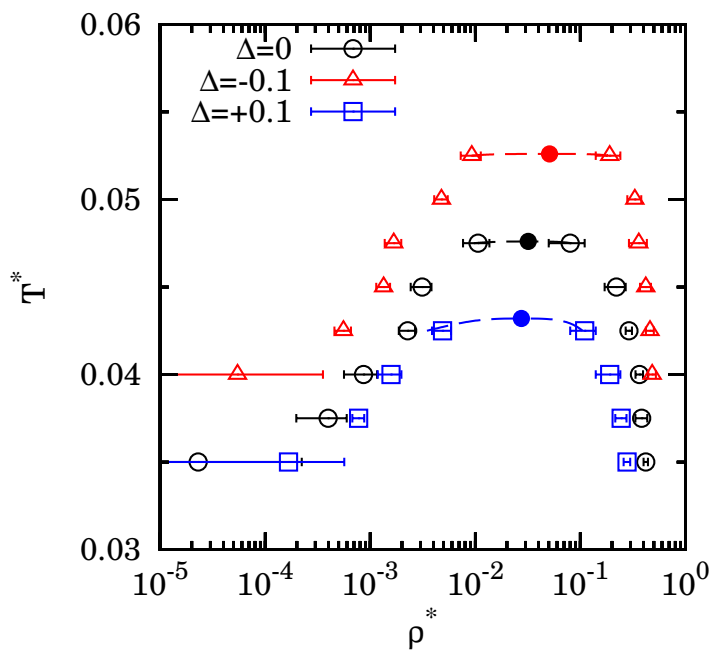

FIG. 11. (Color online) Binodals obtained with the GEMC simulations. The dashed lines are the result of the extrapolation described in the text. The filled circles are the critical points.

$\rho_{c}^{*}=0.0275, T_{c}^{*}=0.0432$, and the RPM with negative nonadditivity, $\Delta=-0.1$, for which $\rho_{c}^{*}=0.0495$, $T_{c}^{*}=0.0526$. We stress that these numbers, in particular the values of critical densities should be considered more as indicative of the dependence of the critical point location on diamater non additivity than as accurate estimates.

We believe that our results can be relevant for the interpretation of experimental work on the phase diagrams of room temperature ionic liquids [42] like the phosphonium halogenide in alkanes solvents and 1-hexyl 3-methyl imidazolium tetrafluoro borate $\left(\mathrm{C}_{6} \mathrm{mimBF}_{4}\right)$ in alcohols and water. The degree of nonadditivity seems directly related to the anion-cation contact-pairing affinity [36]. The salts in the (hydrocarbon) solution dissociate in cations (the phosphonium) and anions (the halogen atoms). The contact affinity between anions and cations is mediated by the solvent and different solvents produce different affinities. As a consequence, in the experimental work of Ref. 42] they observe liquid-liquid coexistence curves which, depending on the kind of solvent used in the ionic liquid mixture, can be above $\left(\mathrm{C}_{6} \mathrm{mimBF}_{4}\right.$ in alcohols and water) the one of the pure RPM theoretical model or below (phosphonium halogenide in alkanes) in reduced units. Moreover, when plotted into a corresponding state representation all the experimental binodals seem to collapse on a same curve even if this occurs very close to the critical point. We then try to see if the law of corresponding states holds or not for our fluid and we find that far from the critical point it is not strictly satisfied, as shown by Fig. 12 Interestingly enough, a plot of the RDF between corresponding states shows an almost complete overlap of the three curves upon a 
TABLE III. Phase coexistence properties for the pure RPM $(\Delta=0)$ and the non-additive $\operatorname{RPM}(\Delta \neq 0)$. $T^{*}$ is the reduced temperature, $N$ is the total number of particles in the system for a certain run, $N_{g}$ is the average number of particles in the gas box during the run, $\mu_{l}^{*}=\mu_{l} \epsilon \sigma / q^{2}-T^{*} \ln \Lambda^{3}$ is the reduced chemical potential of the liquid box $(\Lambda$ being the de Broglie thermal wavelength), $U_{i}^{\text {ex }}$ is the total excess internal energy, and $\rho_{i}^{*}$ is the reduced density, of the gas phase $i=g$ and the liquid phase $i=l$.

\begin{tabular}{|c|c|c||c|c||c|c||c|c|}
\hline$\Delta$ & $T^{*}$ & $N$ & $N_{g} / N$ & $-\mu_{l}^{*}$ & $-U_{g}^{\text {ex }} / N$ & $-U_{l}^{\text {ex }} / N$ & $\rho_{g}^{*}$ & $\rho_{l}^{*}$ \\
\hline 0 & 0.0475 & 370 & $0.51(1)$ & $0.63(1)$ & $0.547(1)$ & $0.609(1)$ & $5.2(9) \times 10^{-3}$ & $0.11(3)$ \\
0 & 0.0475 & 200 & $0.33(1)$ & $0.63(1)$ & $0.559(2)$ & $0.604(1)$ & $1.1(3) \times 10^{-2}$ & $0.08(3)$ \\
0 & 0.045 & 370 & $0.26(1)$ & $0.69(3)$ & $0.528(4)$ & $0.6400(7)$ & $2.3(5) \times 10^{-3}$ & $0.22(5)$ \\
0 & 0.045 & 100 & $0.27(1)$ & $0.63(2)$ & $0.537(4)$ & $0.6393(9)$ & $3.1(7) \times 10^{-3}$ & $0.22(5)$ \\
0 & 0.0425 & 100 & $0.166(8)$ & $0.65(1)$ & $0.52(1)$ & $0.6576(8)$ & $2.3(4) \times 10^{-3}$ & $0.29(2)$ \\
0 & 0.04 & 100 & $0.069(5)$ & $0.73(1)$ & $0.50(2)$ & $0.6745(5)$ & $8(3) \times 10^{-4}$ & $0.35(3)$ \\
0 & 0.0375 & 100 & $0.036(2)$ & $0.72(1)$ & $0.4(1)$ & $0.6835(5)$ & $4(2) \times 10^{-4}$ & $0.38(5)$ \\
0 & 0.035 & 100 & $0.0020(6)$ & $0.75(2)$ & $0.05(40)$ & $0.6938(5)$ & $2(20) \times 10^{-5}$ & $0.42(2)$ \\
\hline-0.1 & 0.0525 & 200 & $0.297(9)$ & $0.71(2)$ & $0.602(2)$ & $0.6844(9)$ & $9(2) \times 10^{-3}$ & $0.19(5)$ \\
-0.1 & 0.05 & 100 & $0.37(1)$ & $0.67(1)$ & $0.609(4)$ & $0.712(1)$ & $4.8(7) \times 10^{-3}$ & $0.33(5)$ \\
-0.1 & 0.0475 & 100 & $0.094(4)$ & $0.71(2)$ & $0.562(7)$ & $0.7240(8)$ & $1.7(3) \times 10^{-3}$ & $0.36(7)$ \\
-0.1 & 0.045 & 100 & $0.092(5)$ & $0.69(2)$ & $0.590(9)$ & $0.7380(8)$ & $1.3(2) \times 10^{-3}$ & $0.42(5)$ \\
-0.1 & 0.0425 & 100 & $0.031(3)$ & $0.85(3)$ & $0.4(1)$ & $0.7503(8)$ & $5.6(10) \times 10^{-4}$ & $0.46(4)$ \\
-0.1 & 0.04 & 100 & $0.0034(7)$ & $0.83(2)$ & $0.08(40)$ & $0.7582(7)$ & $5(30) \times 10^{-5}$ & $0.48(4)$ \\
\hline+0.1 & 0.0425 & 100 & $0.40(2)$ & $0.58(1)$ & $0.493(3)$ & $0.5620(9)$ & $5(1) \times 10^{-3}$ & $0.11(3)$ \\
+0.1 & 0.04 & 100 & $0.23(1)$ & $0.62(3)$ & $0.487(2)$ & $0.5877(9)$ & $1.6(4) \times 10^{-3}$ & $0.19(5)$ \\
+0.1 & 0.0375 & 100 & $0.068(5)$ & $0.70(2)$ & $0.40(4)$ & $0.6068(5)$ & $8(1) \times 10^{-4}$ & $0.24(3)$ \\
+0.1 & 0.035 & 100 & $0.015(2)$ & $0.72(2)$ & $0.17(26)$ & $0.6157(6)$ & $1.7(40) \times 10^{-4}$ & $0.28(2)$ \\
\hline
\end{tabular}

shift by $\pm \Delta$ in $r$, as is show by Fig 13 We think that the only visible difference, the contact values of the like $\mathrm{RDF}$, is a direct hallmark of the break-up of the corresponding states, as physical consequence of the existence of a third relevant interaction parameter, in addition to the unlike hard-sphere diameter and the electric charge. While the $\mathrm{Cl}^{-}$ion and the $\mathrm{BF}_{4}^{-}$anion may reasonably

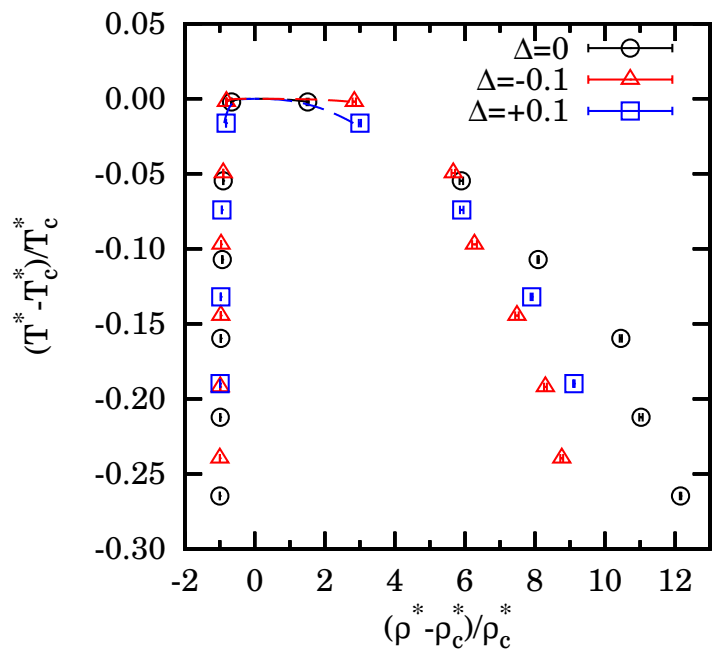

FIG. 12. (Color online) Corresponding state representation of the phase diagram.

well be approximated by a sphere so that the center of charge is identical with the center of mass, the $\mathrm{NTF}_{2}^{-}$anion is by no means spherical. The $\mathrm{NTF}_{2}^{-}$anion is flexible and allows for different conformers. The nitrogen atom in the anion is not necessary identical with the center of mass and the center of charges [43]. In these cases instead of the RPM it is better to choose the PM with ions of differing sizes as reference system [31 33].

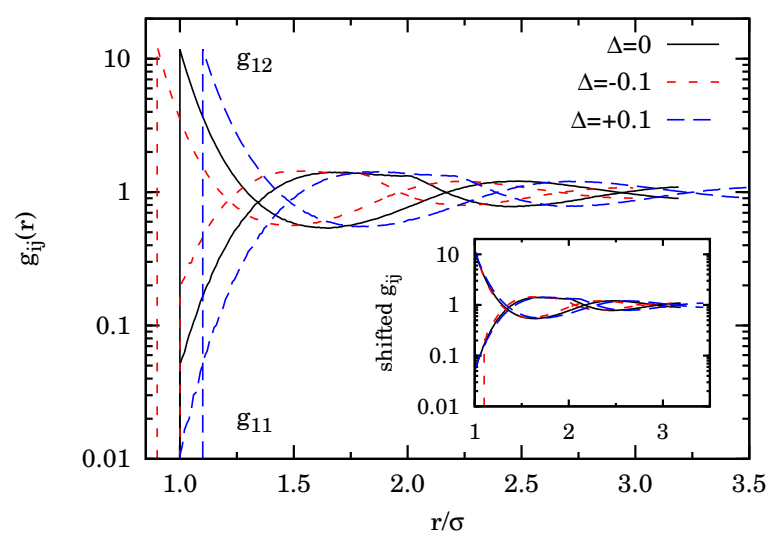

FIG. 13. (Color online) RDF on corresponding states of the liquid branch at $T^{*}=0.0375, \rho^{*}=0.38, \Delta=0, T^{*}=$ $0.035, \rho^{*}=0.28, \Delta=+0.1$, and $T^{*}=0.0425, \rho^{*}=0.46, \Delta=$ -0.1 . In the inset are shown the functions shifted by $\pm \Delta$. 


\section{THEORETICAL REMARKS ON THE CLUSTERING}

Under highly diluted conditions [58] we can approximate the fluid as an ideal mixture of cation-anions, anions, and cations with partial densities $\rho_{ \pm}=(1-\alpha) \rho / 2$, $\rho_{-}=\rho_{+}=\alpha \rho / 2$, respectively, and for the chemical potentials $\mu_{ \pm}=k_{B} T \ln \left((1-\alpha) \rho \Lambda_{+}^{3} \Lambda_{-}^{3} / 2 K\right), \mu_{-}=$ $k_{B} T \ln \left(\alpha \rho \Lambda_{+}^{3} / 2\right)$, and $\mu_{+}=k_{B} T \ln \left(\alpha \rho \Lambda_{-}^{3} / 2\right)$, where $\Lambda_{-}$ and $\Lambda_{+}$are the de Broglie thermal wavelengths of the anions and cations respectively. $K$ is the configurational integral of a cation-anion pair

$$
K=4 \pi \int_{\sigma(1+\Delta)}^{r_{c}} r^{2} e^{\lambda_{B} / r} d r
$$

where $\lambda_{B}=\sigma / T^{*}$ is the Bjerrum length and $r_{c}$ is a cutoff radius conventionally chosen equal to $\lambda_{B} / 2$ corresponding to the minimum of the integrand. At equilibrium $\mu_{ \pm}=\mu_{+}+\mu_{-}$, which implies $(1-\alpha) / \alpha^{2}=K \rho / 2$. Solving for $\alpha$ yields

$$
\alpha=\frac{\sqrt{1+2 K \rho}-1}{K \rho},
$$

An approximate closed form expression for $K$ valid at low $T^{*}$ can be obtained by writing for the anion-cation pair distance $r=\sigma(1+\Delta)+\delta r$ with $\delta r$ small. Then $\sigma / r \approx$ $1 /(1+\Delta)-\sigma \delta r / \sigma^{2}(1+\Delta)^{2}=2 /(1+\Delta)-r / \sigma(1+\Delta)^{2}$. Substituting into Eq. (4.1) and performing the integral with $r_{c}=\infty$ yields

$$
\begin{aligned}
K \approx & 4 \pi \sigma^{3}(1+\Delta)^{4} e^{1 / T^{*}(1+\Delta)} T^{*} \\
& \times\left\{1+2(1+\Delta) T^{*}\left[1+(1+\Delta) T^{*}\right]\right\} .
\end{aligned}
$$

In our simulations we are never in this very diluted condition and as a consequence we observe the formation of clusters of an higher number of particles than just the dimers. So to estimate the cluster concentrations $x_{n}^{c}=\left\langle N_{n}\right\rangle / N$, we need a different analysis closer in spirit to the one of Tani and Henderson [45, 59]. Simplifying that analysis we can consider as the inter-cluster configurational partition function the one of an ideal gas of clusters, in reduced units, $Z_{\text {inter }} \approx\left(V / \sigma^{3}\right)^{N_{t}}$, where $N_{t}=\sum_{n=1}^{n^{c}} N_{n}$ is the total number of clusters and we assume to have only clusters made of up to $n^{c}$ particles. Then the equations for the equilibrium cluster concentrations $x_{n}^{c}$ are

$$
\begin{aligned}
x_{n}^{c} & =\lambda^{n} z_{n}^{\text {intra }} / \rho^{*}, \quad n=1,2, \ldots, n^{c}, \\
1 & =\sum_{n=1}^{n^{c}} n x_{n}^{c},
\end{aligned}
$$

where $z_{n}^{\text {intra }}$ are the configurational intra-cluster partition functions in reduced units with $z_{1}^{\text {intra }}=2$ and $\lambda\left(=\alpha \rho^{*} / 2\right)$ is a Lagrange multiplier. Moreover, neglecting the excess internal energy of the clusters we can approximate $z_{n}^{\text {intra }} \approx\left(v_{n} / \sigma^{3}\right)^{n-1} \sum_{s=0}^{n}(s !(n-s) !)^{-1}=$ $\left(v_{n} / \sigma^{3}\right)^{n-1} 2^{n} / n$ ! where $v_{n}$ is the volume of an $n$-cluster.
Moreover, assuming further the cluster to be in a closed packed configuration we can approximate, for $\Delta=0$, $v_{n} \approx n \sigma^{3} / \sqrt{2}$. Notice that for $\Delta \neq 0$ we would expect $v_{n}$ to change by a constant multiplicative factor which would still give the same result for the cluster concentrations. Clearly a proper analysis of the $n$-cluster volume would require a MC simulation [7]. This temperature independent approximation gives for $n^{c}=100$ the results shown in Fig. 14 (note that the results have very small dependence on $\left.n^{c}\right)$.

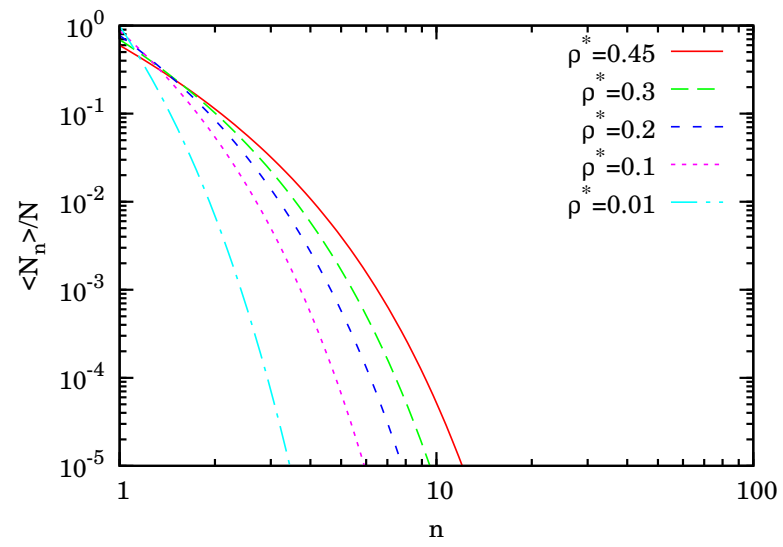

FIG. 14. (Color online) Cluster analysis described in the text for $n^{c}=100$ at various densities.

Form the figure we can say that our simulation results for $T^{*}=0.1$ and $\Delta=-0.3$ have qualitatively the same behavior of our oversimplified analysis. This justifies the fit of Table I where the Laplace multiplier is considered as a fitting parameter. The strong dependence from the nonadditivity (and on temperature) that we observe in the simulation is an indication that the approximation of neglecting the excess internal energy of a cluster is too severe. One should consider $z_{n}^{\text {intra }}=e^{-n f_{n}^{\text {ex }} / T^{*}}\left(v_{n} / \sigma^{3}\right)^{n-1} 2^{n} / n$ !, where $f_{n}^{\operatorname{ex}}\left(T^{*}\right)=$ $\int_{0}^{1 / T^{*}} u_{n}^{\mathrm{ex}}(1 / x) d x$ is the excess free energy per particle of the $n$-cluster and $u_{n}^{\mathrm{ex}}\left(T^{*}\right)=\left(\epsilon \sigma / q^{2}\right)\left\langle\sum_{i>j=1}^{n} \phi_{i j}\left(r_{i j}\right)\right\rangle / n$ is the reduced excess internal energy per particle of the $n$-cluster. Note once again that choosing an $f_{n}^{\text {ex }}$ independent of $n$ would lead to the same oversimplified result we described for the cluster concentrations. What really matters is the combined dependence of $f_{n}^{\operatorname{ex}}\left(T^{*}\right)$ on $n$ and $T^{*}$ which can be assessed within the MC simulation 7, 45. For example the curves of Figs. 2 and 3 with percolating clusters are better fitted by the following three parameters expression $x_{n}^{c} \approx \lambda^{n+a n^{2}} n^{b n} / n !$.

One thing that can be done is to distinguish amongst the clusters of $n$ particles between the ones formed by $s$ negative particles and $t$ positive particles with $t+s=n$, as done in Ref. 25], in order to be able to approximate analytically the intra-cluster excess free energy per par- 
ticle

$$
\begin{aligned}
z_{n}^{\text {intra }}= & \sum_{s=0}^{n} z_{s, n-s}^{\text {intra }}, \\
z_{s, t}^{\text {intra }}= & \frac{1}{s ! t !} \frac{1}{\sigma^{3(s+t-1)}} \int_{\Omega_{s, t}} d \mathbf{r}_{2} \ldots d \mathbf{r}_{s+t} \\
& \times e^{-\beta \sum_{\mu>\nu=1}^{s+t} \phi_{i_{\mu} j_{\nu}}\left(r_{\mu \nu}\right)},
\end{aligned}
$$

where the configurational integral goes only over the relative positions and it covers the region $\Omega_{s, t}$ of cluster configuration space. This way one can quantitatively 25] estimate how the Tani and Henderson theory [59] deviates from the exact $\mathrm{MC}$ results.

We immediately see how $z_{1,1}^{\text {intra }} \propto K / \sigma^{3}$ becomes bigger and bigger as $\Delta \rightarrow-1$ and the same holds for all the $z_{k, k}^{i n t r a}$ which clearly dominate over all the others $z_{s, t}^{\text {intra }}$ with $s \neq t$. This qualitatively explains the Fig. [4 as is shown in Fig. [15] where we show the results from the approximation described in Appendix A for $n^{c}=30, T^{*}=0.1, \rho^{*}=0.45, a=1.5, b=0.9$ and various values of $\Delta$ (note that the results have very small dependence on $n^{c}$ ).

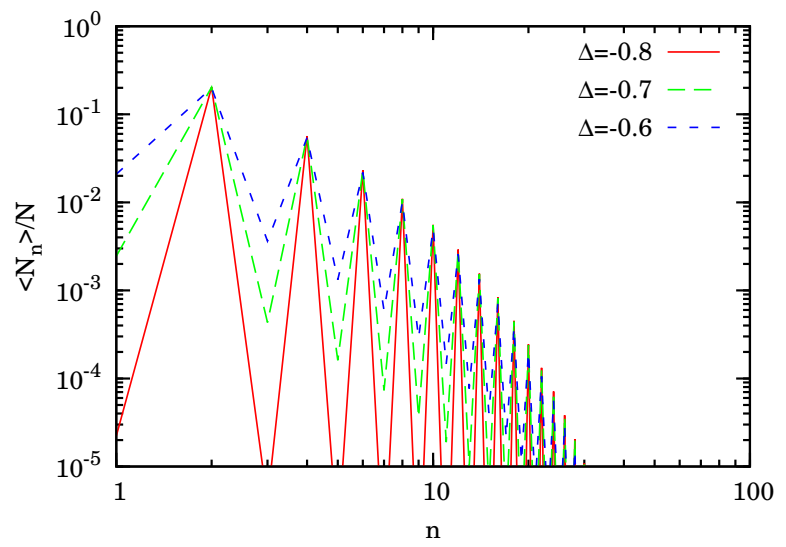

FIG. 15. (Color online) Cluster analysis described in the text for $n^{c}=30, T^{*}=0.1, \rho^{*}=0.45, a=1.5, b=0.9$ at various values of $\Delta$.

\section{CONCLUSIONS}

In conclusion we have performed NVT MC simulations of the RPM with non-additive hard-sphere diameters outside the coexistence region with particular emphasis on the clustering properties. In order to establish whether the cluster analysis falls outside the gas-liquid coexistence region for a given value of the nonadditivity we accurately determined the binodals of the nonadditive fluid using the Gibbs ensemble method after a density distribution function analysis to get a first insights on the shifts of the coexistence region with the nonadditivity. It turned out that a negative nonadditivity tends to shift to higher temperatures and higher densities the critical point whether a positive one shifts it to lower temperatures and densities. The law of corresponding states does not seem to be strictly fulfilled over an extended region below the critical point for $\Delta=0, \pm 0.1$. Our results can be used as a theoretical support to the analysis of experimental work on room temperature ionic liquids 41 43] where shifts in the liquid-liquid binodals akin to ours are observed as a function of the kind of solvent used in the ionic mixture.

From the cluster analysis, we were able to distinguish between two kind of behaviors for the cluster concentrations. When we do not observe percolating clusters during the simulation, the curves for the cluster concentrations as a function of the cluster size are independent of the number of particles used in the simulation. When we observe percolation during the simulation the curves depend on the number of particles used in the simulation but obey a straightforward scaling with $N$ relationship.

At low densities the negative non-additive fluid has stronger clustering than the pure RPM whereas at high densities is the positive non-additive fluid to have the strongest one. The positive non-additive fluid is the first one reaching the percolating clusters upon an increase of density. This certainly depends on the fact that for a positive nonadditivity the ions have less space where to move at a given density and, due to he presence of two opposite charged species, there is a competition between the tendency to clustering driven by the Coulomb interaction and tendency to demixing due to entropic reasons. A negative nonadditivity tends to favor the formation of the neutrally charged clusters starting with dipolar ones. Traces of these features can also be read from an analysis of the partial radial distribution function and structure factors. Our clustering results can be summarized observing that at high density for a positive deviations from additivity we have more clustering than in the additive model whereas for a negative deviation from additivity we have less clustering than in the additive model. At low density the reverse behavior is found. These results can be explained by the following arguments: at high density a positive nonadditivity leaves less effective volume to the particles and a negative nonadditivity leaves more effective volume relative to the additive model; at low density a negative nonadditivity favors the formation of neutral clusters and a positive nonadditivity favors the competition between the tendency to demixing in a neutral mixture and the tendency to microscopic intermixing of the two species favored by the Coulombic interactions. These observations are in agreement with the fact that the energy of a cation-anion pair at contact increases for positive nonadditivity and decreases for negative nonadditivity.

A simple, temperature independent, clustering theory where we regard the clusters as forming an ideal gas and we approximate the $n$-cluster as an ideal ensemble of $n$ particles in a closed packed configuration can be used to 
qualitatively explain the cluster concentrations observed at not to high density and absolute value of the nonadditivity. In order to qualitatively explain the prevalence of the neutral clusters in the negatively non-additive fluid it is necessary to refine the approximation at the intracluster level.

In the future it would be desirable to make quantitative the comparison between clustering theory and $\mathrm{MC}$ exact results. It would be also interesting the determination of the percolation threshold as a function of nonadditivity. In the temperature density phase diagram, one can determine the percolation threshold calculating the fraction of configurations with percolating clusters within the NVT simulation as a function of density for two systems of different size $N$. A point of the percolation threshold results then from where the curves of the two systems meet.

\section{Appendix A: Approximated intracluster configurational partition function for negative nonadditivity}

Let us call the anions $i_{-}=1_{-}, \ldots, s_{-}$and the cations $j_{+}=1_{+}, \ldots, t_{+}$. From Eq. (4.7) in the main text follows

$$
\begin{aligned}
z_{t, t}^{i n t r a}= & \frac{1}{t !^{2}} \frac{1}{\sigma^{3(2 t-1)}} \int_{\Omega_{t, t}} \prod_{l=2}^{t} d \mathbf{r}_{1_{+} l_{-}} \prod_{k=1}^{t} d \mathbf{r}_{k_{+} k_{-}} \\
& \times \prod_{i>j=1}^{t} e^{-2 \lambda_{B} / r_{i_{+} j_{+}}} \prod_{i, j=1}^{t} e^{+\lambda_{B} / r_{i_{+} j_{-}}} \\
\approx & \frac{1}{t !^{2}} \frac{1}{\sigma^{3(2 t-1)}} \int_{\Omega_{t, t}} \prod_{l=2}^{t} d \mathbf{r}_{1_{+} l_{-}} \prod_{k=1}^{t} d \mathbf{r}_{k_{+} k_{-}} \\
& \times \prod_{i, j=1}^{t} e^{+\lambda_{B} / r_{i_{+} j_{-}}}
\end{aligned}
$$

where we approximated $e^{-\lambda_{B} / r} \approx 1$ which is justified at high $T^{*}<1 / 2(1+\Delta)$ or low $\lambda_{B}$. Now we observe that for example $r_{1_{+} 2_{-}}=\left|\mathbf{r}_{1_{+} 1_{-}}+\mathbf{r}_{1_{-} 2_{-}}\right|$with $r_{1_{-} 2_{-}}>\sigma$ and $e^{+\lambda_{B} / r_{1}{ }^{2}-} \approx 1$. So that for negative nonadditivity we can further approximate

$$
\begin{aligned}
z_{t, t}^{\text {intra }} \approx & \frac{1}{t !^{2}} \frac{1}{\sigma^{3(2 t-1)}} \int_{\Omega_{t, t}} \prod_{l=2}^{t} d \mathbf{r}_{1_{+} l_{-}} \prod_{k=1}^{t} d \mathbf{r}_{k_{+} k_{-}} \\
& \times \prod_{i, j=1}^{t} e^{+\lambda_{B} / r_{i_{+} j_{-}}} \\
\approx & \frac{1}{t !^{2}} \frac{1}{\sigma^{3(2 t-1)}} \int_{\Omega_{t, t}} \prod_{l=2}^{t} d \mathbf{r}_{1_{+} l_{-}} \prod_{k=1}^{t} d \mathbf{r}_{k_{+} k_{-}} \\
& \times \prod_{i=1}^{t} e^{+\lambda_{B} / r_{i_{+} i_{-}}} \\
\propto & \frac{(2 t)^{b(2 t-1)}}{t !^{2}}\left(K / K_{0}\right)^{t},
\end{aligned}
$$

where the factor $(2 \dot{t})^{b(2 t-1)}$ takes into account the volume of $\Omega_{t, t}$ with $b$ a free parameter and

$$
K / K_{0}=\int_{a \sigma(1+\Delta)}^{\lambda_{B} / 2} r^{2} e^{+\lambda_{B} / r} d r / \int_{a \sigma(1+\Delta)}^{\lambda_{B} / 2} r^{2} d r
$$

with $a$ a second free parameter. With the same approximations we can say

$$
z_{s, t}^{i n t r a} \stackrel{\propto}{\sim} \frac{(s+t)^{b(s+t-1)}}{s ! t !}\left(K / K_{0}\right)^{\min \{s, t\}}
$$

\section{ACKNOWLEDGMENTS}

R.F. would like to acknowledge the use of the computational facilities of CINECA through the ISCRA call. The GEMC code took 26 min of CPU time for 10 million steps of a system of size $N=200$ on the IBM PLX (iDataPlex DX360M3) cluster.
[1] D. Henderson, M. Holovko, and A. Trokhymchuk, ed., Ionic Soft Matter: Modern Trends in Theory and Applications, vol. 206 of NATO Science series (Springer, Netherlands, 2004).

[2] P. G. de Gennes, Rev. Mod. Phys. 64, 645 (1992).

[3] R. Fantoni and G. Pastore, J. Chem. Phys. 120, 10681 (2004).

D. Gazzillo, A. Giacometti, R. Fantoni, and P. Sollich, Phys. Rev. E 74, 051407 (2006).

R. Fantoni, A. Giacometti, A. Malijevský, and A. Santos, J. Chem. Phys. 133, 024101 (2010).

R. Fantoni, A. Giacometti, F. Sciortino, and G. Pastore, Soft Matter 7, 2419 (2011).

R. Fantoni, Eur. Phys. J. B 85, 108 (2012).

R. Fantoni, J. W. O. Salari, and B. Klumperman, Phys.
Rev. E 85, 061404 (2012).

[4] J. P. Hansen and I. R. McDonald, Theory of Simple Liquids (Academic Press, 1986), 2nd ed.

[5] G. Stell, K. C. Wu, and B. Larsen, Phys. Rev. Lett. 37, 1369 (1976).

[6] T. L. Croxton and D. A. McQuarries, J. Phys. Chem. 83, 1840 (1979).

[7] M. J. Gillan, Mol. Phys. 49, 421 (1983).

[8] T. Cartailler, P. Turq, L. Blum, and N. Condamine, J. Phys. Chem. 96, 6766 (1992).

[9] J. A. Given, Phys. Rev. A 45, 3849 (1992).

[10] J. A. Given and G. Stell, J. Chem. Phys. 96, 9233 (1992).

[11] M. E. Fisher and Y. Levin, Phys. Rev. Lett. 71, 3826 (1993).

[12] M. E. Fisher, J. Stat. Phys. 75, 1 (1994). 
[13] G. Stell, J. Stat. Phys. 78, 197 (1995).

[14] Y. Zhou, S. Yeh, and G. Stell, J. Chem. Phys. 102, 5785 (1995).

[15] S. G. Yeh, Y. Q. Zhou, and G. Stell, J. Phys. Chem. 100, 1415 (1996).

[16] J. A. Given and G. Stell, J. Chem. Phys. 106, 1195 (1997).

[17] J. Jiang, L. Blum, O. Bernard, J. M. Prausnitz, and S. I. Sandler, J. Chem. Phys. 116, 7977 (2002).

[18] H. L. Friedman and B. Larsen, J. Chem. Phys. 70, 92 (1979).

[19] P. N. Vorontsov-Veliaminov, A. M. Elỳashevich, L. A. Morgenshtern, and V. P. Chasovshikh, High. Temp. (USSR) 8, 261 (1976).

[20] V. P. Chasovshikh and P. N. Vorontsov-Veliaminov, High. Temp. (USSR) 14, 174 (1976).

[21] A. Z. Panagiotopoulos, Fluid. Phase Equil. 76, 97 (1992).

[22] I. S. Graham and J. P. Valleau, J. Phys. Chem. 94, 7894 (1990).

[23] J.-M. Caillol, J. Chem. Phys. 100, 2161 (1994).

[24] G. Orkoulas and A. Z. Panagiotopoulos, J. Chem. Phys. 101, 1452 (1994).

[25] J.-M. Caillol and J.-J. Weis, J. Chem. Phys. 102, 7610 (1995).

[26] G. Orkoulas and A. Z. Panagiotopoulos, J. Chem. Phys. 110, 1581 (1999).

[27] Q. Yan and J. J. de Pablo, J. Chem. Phys. 111, 9509 (1999).

[28] E. Luijten, M. E. Fisher, and A. Z. Panagiotopoulos, Phys. Rev. Lett. 88, 185701 (2002).

[29] J.-M. Caillol, D. Levesque, and J.-J. Weis, J. Chem. Phys. 116, 10794 (2002).

[30] P. J. Camp and G. N. Patey, J. Chem. Phys. 111, 9000 (1999).

[31] J. M. Romero-Enrique, G. Orkoulas, A. Z. Panagiotopoulos, and M. E. Fisher, Phys. Rev. Lett. 85, 4558 (2000).

[32] A. Z. Panagiotopoulos and M. E. Fisher, Phys. Rev. Lett. 88, 045701 (2002).

[33] Q. Yan and J. J. de Pablo, Phys. Rev. Lett. 86, 2054 (2001).

[34] Q. Yan and J. J. de Pablo, Phys. Rev. Lett. 88, 095504 (2002).

[35] G. Pastore, P. V. Giaquinta, J. S. Thakur, and M. P. Tosi, J. Chem. Phys. 84, 1827 (1986), The relationship between our reduced units and theirs is as follows: $\rho^{*}=$ $3 \sigma^{3} / 4 \pi$ and $T^{*}=\sigma / \Gamma$.

[36] I. Kalcher, J. C. F. Schulz, and J. Dzubiella, Phys. Rev. Lett. 104, 097802 (2010).

[37] M. Rovere and G. Pastore, J. Phys.: Condens. Matter 6, A163 (1994).

E. Lomba, M. Alvarez, L. L. Lee, and N. G. Almarza, J. Chem. Phys. 104, 4180 (1996).

K. Jagannathan and A. Yethiraj, J. Chem. Phys. 118, 7907 (2003).

W. T. Góźdź, J. Chem. Phys. 119, 3309 (2003).

A. Buhot, J. Chem. Phys. 122, 024105 (2005).

A. Santos, M. López de Haro, and S. B. Yuste, J. Chem. Phys. 132, 204506 (2010).

[38] R. Fantoni and G. Pastore, Europhys. Lett. (2013), in press.

[39] W. C. K. Poon, S. U. Egelhaaf, J. Stellbrink, J. Allgaier, A. B. Schofield, and P. N. Pusey, Phil. Trans. R. Soc. Lond. A 359, 897 (2001).

W. C. K. Poon, J. Phys.: Condens. Matter 14, R859
(2002).

[40] H. Weingärtner, M. Kleemeier, S. Wiegand, and W. Schöer, J. Stat. Phys. 78, 169 (1995).

[41] M. Kleemeier, S. Wiegand, W. Schröer, and H. Weingärtner, J. Chem. Phys. 110, 3085 (1999).

[42] D. Saracsan, C. Rybarsch, and W. Schröer, Z. Phys. Chem. 220, 1417 (2006).

[43] W. Schröer and V. R. Vale, J. Phys.: Condens. Matter 21, 424119 (2009).

[44] M. P. Allen and D. J. Tildesley, Computer Simulation of Liquids (Oxford University Press, 1987).

[45] R. Fantoni, A. Giacometti, F. Sciortino, nd G. Pastore, Soft Matter 7, 2419 (2011). R. Fantoni, Eur. Phys. J. B 85, 108 (2012).

[46] Many different ways of defining a cluster have been proposed [7, 11, 60 62], since the Bjerrum theory [63] of ionic associations first appeared. Our choice corresponds to the one of Gillan [7] and Caillol and Weis [25].

[47] L. Rovigatti, J. Russo, and F. Sciortino, Phys. Rev. Lett. 107, 237801 (2011).

[48] A. B. Bhatia and D. E. Thornton, Phys. Rev. B 2, 3004 (1970).

[49] R. Fantoni, D. Gazzillo, and A. Giacometti, Phys. Rev. E 72, 011503 (2005).

[50] M. Rovere, D. W. Heermann, and K. Binder, Europhys. Lett. 6, 585 (1988).

M. Rovere, D. W. Heermann, and K. Binder, J. Phys.: Condens. Matter 2, 7009 (1990).

M. Rovere, P. Nielaba, and K. Binder, Z. Phys. B 90, 215 (1993).

[51] C. Vega, J. L. F. Abascal, C. McBride, and F. Bresme, J. Chem. Phys. 119, 964 (2003).

[52] D. Frenkel and B. Smit, Understanding Molecular Simulation (Academic Press, San Diego, 1996).

A. Z. Panagiotopoulos, Mol. Phys. 61, 813 (1987).

A. Z. Panagiotopoulos, N. Quirke, M. Stapleton, and D.

J. Tildesley, Mol. Phys. 63, 527 (1988).

B. Smit, Ph. De Smedt, and D. Frenkel, Mol. Phys. 68, 931 (1989).

B. Smit and D. Frenkel, Mol. Phys. 68, 951 (1989).

[53] J. V. Sengers and J. M. H. Levelt-Sengers, in Progress in Liquid Physics, edited by C. A. Croxton (Wiley, Chichester, 1978), chap. 4.

[54] F. Wegner, Phys. Rev. B 5, 4529 (1972).

[55] J.-M. Caillol, D. Levesque, and J. J. Weis, Phys. Rev. Lett. 77, 4039 (1996).

[56] J.-M. Caillol, Condensed Matter Physics 7, 741 (2004).

[57] J. V. Sengers and J. G. Shanks, J. Stat. Phys. 137, 857 (2009).

[58] C. Valeriani, P. J. Camp, J. W. Zwanikken, R. van Roij, and M. Dijkstra, Soft Matter 6, 2793 (2010).

[59] A. Tani and D. Henderson, J. Chem. Phys. 79, 2390 (1983)

[60] J. K. Lee, J. A. Barker, and F. F. Abraham, J. Chem. Phys. 58, 3166 (1973).

[61] W. Ebeling and M. Grigo, Am. Phys. 37, 21 (1980).

[62] H. L . Friedman and G. Larsen, J. Chem. Phys. 70, 92 (1979).

[63] N. Bjerrum, Kgl. Dan. Vidensk. Selsk. Mat.-fys. Medd. 7, 1 (1926). 